ThE Astrophysical JournaL, 318:215-231, 1987 July 1

(C) 1987. The American Astronomical Society. All rights reserved. Printed in U.S.A.

\title{
THE DISTANCE TO M5 FROM ITS RR LYRAE VARIABLES
}

\author{
J. G. COHEN AND G. A. Gordon \\ Palomar Observatory, California Institute of Technology \\ Received 1986 November 7; accepted 1986 December 17
}

\begin{abstract}
We have applied a variation of the Baade-Wesselink method to four RR Lyrae variables in the moderately metal-poor globular cluster M5. The radial velocity curves are from observations with the 200 inch $(5 \mathrm{~m}) \mathrm{Hale}$ telescope, while the photometry is derived from CCD images through a Johnson $B$ and a near-infrared $i$ filter. The method developed for the analysis utilizes an effective wavelength for each filter and relies on the accuracy of the calibration of absolute flux for bright standard stars by Oke and Gunn, as well as on the accuracy of the absolute emitted fluxes predicted in the near-infrared by the Kurucz grid of model stellar atmospheres.

The results must be viewed as preliminary thus far due to apparent discrepancies between the photometrically and spectroscopically deduced angular diameter-phase relationships which are most apparent in the part of the cycle immediately following maximum light. This restricts the range of phase that can be used to derive the distance to the variables. The best value we obtain for the mean of the 4 RR Lyrae stars in M5 is an absorption-corrected intensity mean absolute $V$ magnitude of $+1.05 \mathrm{mag}(+0.15,-0.25 \mathrm{mag})$.
\end{abstract}

Subject headings: clusters: globular — stars: RR Lyrae

\section{INTRODUCTION}

The distances to globular clusters are usually determined by fixing the absolute magnitude of their horizontal-branch stars at the location of the RR Lyrae variable gap. The assumed value for the absolute luminosity of RR Lyrae variables rests upon statistical parallaxes for field RR Lyrae stars and on variations of the Baade-Wesselink (Baade 1926; Wesselink 1969) method for field RR Lyrae stars. Distances to globular clusters which rely on main-sequence locations as defined by field subdwarfs such as in Sandage (1970) or Carney (1980) suffer from the serious limitation of the extremely small number of field subdwarfs which have accurate parallaxes, and of the need to include corrections to the luminosity for varying metallicities. Many of the field subdwarfs may be slightly evolved, and thus have higher somewhat luminosities than the true main sequence.

These cluster distances are then used to determine globular cluster ages, and small errors in distance propagate into substantial age uncertainties. In addition, since RR Lyrae stars have been detected in the field and clusters of the Magellanic Clouds (Graham 1984; Graham and Nemec 1984) and in M31 (Pritchet and van den Bergh 1986) from the ground, and will undoubtedly be found in additional galaxies by the Hubble Space Telescope, their absolute magnitudes directly calibrate the extragalactic distance scale. They also serve to determine the distance to the Galactic center (Oort and Plaut 1975).

In recent years, major efforts have been undertaken by several groups to improve the absolute magnitude determinations for field RR Lyrae variables. These include the reanalysis of the statistical parallax problem with improved astrometric data by two independent groups (Hawley et al. 1986; Barnes and Hawley 1986; Strugnell, Reid, and Murray 1986). Manduca and Bell (1981), Manduca et al. (1981), Carney and Latham (1984), Jones et al. (1987a,b), Burki and Meylan (1986a, b), Longmore et al. (1985), Jameson (1986), and Cacciari (1986) have explored variations of the Baade-Wesselink technique. Major surveys are now underway to accumulate observational data which will allow such determinations of distances for statistically significant samples of field RR Lyrae stars over a range of metallicity. While several unresolved problems remain in the analysis of the field variables, substantial progress has been made.

It is clear that the efficiency of modern detectors allows the same techniques to be applied to RR Lyrae variables in globular clusters, and that is the goal of the present effort. A natural candidate is the globular cluster M5. It has low interstellar reddening, about 100 RR Lyrae variables (Hogg 1973), a recent analysis of the periods of the M5 variables by Coutts and Sawyer-Hogg (1969), is relatively nearby so that the variables are as bright as possible, and is accessible from a northern site. In $\S$ II we present the extensive observational material we have accumulated for the RR Lyrae variables in M5 during the 1985 and 1986 observing season. The technique of analysis that we have developed is described in $\S$ III. Section IV $a$ begins with a description of the results of this analysis and then continues in $\S \mathrm{IV} b$ with a comparison to recent results for field stars. A summary of our conclusions is given in the final section.

\section{OBSERVATIONAL MATERIAL FOR THE M5 RR LYRAES}

\section{a) Radial Velocities}

The RR Lyrae variables in M5 were identified from the chart in Bailey (1917). The periods for the variables were taken directly from Hogg (1973). Because the data were taken over a range of time which for two stars was less than 1 month, for one variable was less than 6 weeks, and at the worst was only 1 year, no adjustments to the periods were necessary. (We did check at various points whether small changes in the adopted periods improved the light or radial velocity curves, but never found any definite indication of that.) The zero of our phase system is taken as the first maximum in light for each variable after Julian day 2,446,536.500 (equivalent to 0 hours UT on 1986 April 16). The fundamental data for the RR Lyrae variables discussed here is given in Table 1, which includes their periods, the zero of our phase system, the maximum and 
TABLE 1

Light Curve Parameters for M5 RR Lyrae Stars

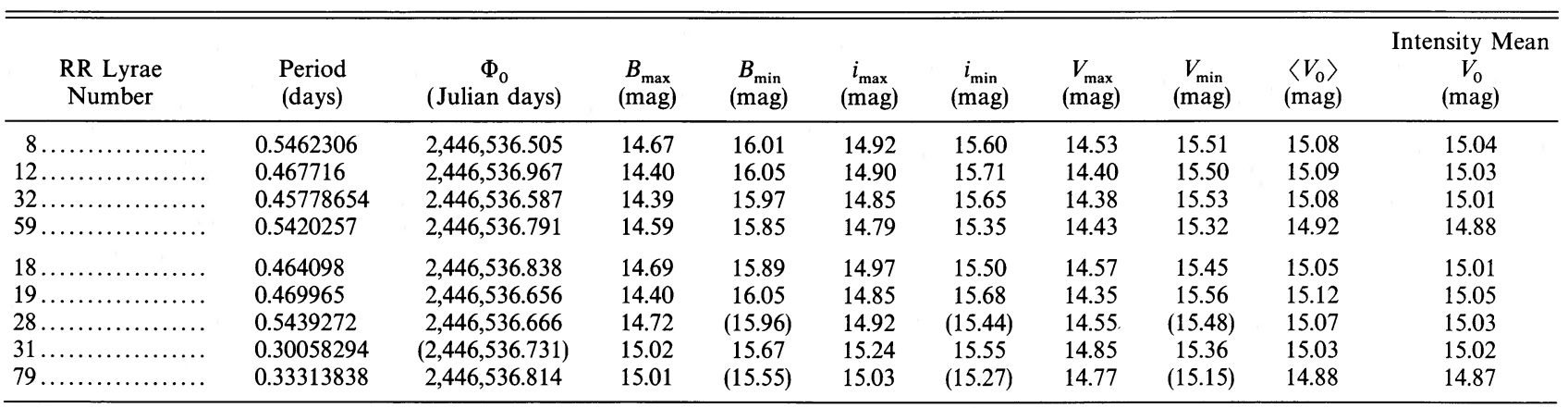

minimum magnitudes in $B, i$, and $V$, and the absorption corrected mean and intensity mean $V$ magnitude (see $\S$ II $b$ ).

The RR Lyrae stars chosen for analysis in M5 included only stars for which Coutts and Sawyer-Hogg (1969) found no evidence for the presence of the Blashko effect. Their extensive photographic archives made it possible for them to identify the presence of even modest cycle-to-cycle variations in several M5 RR Lyrae stars. We saw no evidence in our data of this effect in any of the four stars in M5 which we analyzed in detail.

The spectroscopic data are from the nights of 1985 April 28 to May 1, 1986 April 15-18 and 1986 May 14-17 at the 200 inch Hale telescope at Palomar Mountain. Integrations were always 30 minutes long as a compromise between avoiding excessive blurring in phase and securing an adequate signal level for high-precision velocities from stellar spectra of lowmetallicity stars with few strong spectral features. Observations were carried out irrespective of seeing, transparency, and airmass (within the range up to 2.1 airmasses and absorption due to clouds of less than $1.5 \mathrm{mag}$ ). During the 1985 nights, only the largest amplitude RR Lyrae M5-32 was observed. The first two nights' spectra were taken at the 36 inch $(91 \mathrm{~cm})$ camera at the coudé focus of the 200 inch $(5 \mathrm{~m})$ Hale telescope using a $800 \times 800 \mathrm{TI} C \mathrm{CD}$ as a detector. The resolution was $0.14 \AA$ per pixel. The spectra covered the range $5150-5260 \AA$, so that the strongest spectral lines are the $\mathrm{Mg}$ triplet. Because the image slicers were not working properly, the signal level was rather low, and for the only remaining usable night in 1985 we switched to the red camera (only) of the Double Spectrograph (again with an $800 \times 800$ TI CCD detector). Although this spectrograph was not designed for high-resolution work, by using it in a nonstandard mode with a $1200 \mathrm{~g} \mathrm{~mm}^{-1}$ grating in second order (with substantial vignetting occurring within the optical train), a spectral resolution of $0.37 \AA$ per pixel can be obtained. With a 1" slit, the FWHM of arc lines is 2 pixels. The spectral coverage extended from 5100 to $5400 \AA$, including the $\mathrm{Mg}$ triplet, and various strong $\mathrm{Fe}$-dominated blends such as $5270 \AA$. Typical count rates were 400 electrons per pixel (near maximum light) at the coudé and about 3000 electrons per pixel (near maximum light) at the Double Spectrograph for conditions near 1" seeing. During this night the spectrograph was rotated so that the slit rotated to a position angle $14^{\circ}$ from the nominal east-west orientation (where flexure is minimized) to include the giant M5-IV-82 (in the numbering system of Arp 1962) as well as M5-32 along the slit. This star was of comparable brightness in the $B$ filter to the M5 RR Lyrae variables at maximum light.

During the first 1986 run, the Double Spectrograph was used in the same configuration, but the spectrograph was rotated so that the slit was $16^{\circ}$ from the east-west line so as to pass through two RR Lyrae variables simultaneously, M5-32 and M5-8. (The Double Spectrograph was designed for use as a long-slit instrument, and with the present CCD detector, the allowed slit length exceeds $2^{\prime}$, but the maximum slit length visible on the television guiding system is about $85^{\prime \prime}$. At least three pairs of RR Lyrae variables can be found within M5 that are less than $85^{\prime \prime}$ apart.) The weather was poor during that run, and a $2^{\prime \prime}$ slit had to be used. The second 1986 run we alternated between a slit position angle which allowed simultaneous spectra of M5-8 and of M5-32 and a second position angle which allowed simultaneous spectra of the variables M5-12 and M5-59. The rotation in position angle between the two settings was $20^{\circ}$. Noble gas arcs were always taken before and after each exposure in the correct slit orientation. These observations were carried out with phase tables at hand to allow optimum choice of the pair of variables to be observed at any time in order to maximize phase coverage over the entire period for the four stars.

On those nights during which the Double Spectrograph was in use, several bright template stars were observed each night, as well as giants in M3 and M92 (exposed to a signal level similar to that expected for the M5 RR Lyrae stars), all of whose radial velocities are well known. Fewer template stars, and no additional globular cluster giants, were observed while the coudé was used.

The data were reduced using the image processing VAX 11/780 computer of the astronomy department at Caltech. After flattening, the single spectrum or pair of spectra (as well as a sky spectrum chosen from a clean region as free of stars as possible along the slit immediately adjacent to each object), was extracted to one dimension. Next we subtracted off the appropriate sky spectrum. The spectra were cross-correlated with the template spectrum for each night. The arcs were also cross-correlated with the arc for the template star, arcs before each exposure and afterward being treated separately. This enabled the construction of a flexure table as a function of time during each night. For the coudé data, the flexure was negligible. It was quite small for the Double Spectrograph data when the slit was left close to its normal east-west orientation (i.e., not altering the slit position angle during the night), but reached significant values (up to 1 pixel at extreme airmasses) during the last run when the slit was rotated repeatedly during the night. The cross-correlation peaks, signal levels, and visual appearance of the Mg triplet of all the spectra were examined, and several radial velocities were rejected as unreliable. In 
general, these were from the beginning or end of the night, when the airmass approached 2.1, and the seeing deteriorated, so the signal level obtained in the maximum 30 minute integration was inadequate. (This was done prior to constructing the radial velocity-phase curves.) The zero point of our radial velocities assumes that the heliocentric radial velocity of $\mu$ Leo is $13.86 \mathrm{~km} \mathrm{~s}^{-1}$ and that of HD 122563 is $-24.7 \mathrm{~km} \mathrm{~s}^{-1}$. (These are the mean values for the modern determinations in Abt and Biggs 1972.)

The radial velocities of the M3 stars were compared with those of Gunn and Griffin (1979), and their repeatability from night to night was also examined. The rms deviation about our mean velocity for the three M3 stars observed repeatedly was 4 $\mathrm{km} \mathrm{s}^{-1}$. The comparison with the published radial velocities revealed differences in our mean versus those of Gunn and Griffin with a total range of only $2.7 \mathrm{~km} \mathrm{~s}^{-1}$ once a constant zero point difference was removed. We also note that the rms variation about the mean of our 13 radial velocity measurements for M5-IV-82 was only $1.2 \mathrm{~km} \mathrm{~s}^{-1}$, although admittedly this star is somewhat brighter than the M5 RR Lyrae stars. We therefore assess the uncertainties of our radial velocities from coudé spectra as $3 \mathrm{~km} \mathrm{~s}^{-1}$, and from the Double Spectrograph observations as $4 \mathrm{~km} \mathrm{~s}^{-1}$.

The heliocentric radial velocities derived from the spectra are listed in Table 2 as a function of Julian day and phase within the period for the 4 RR Lyrae variables in M5. The time and phase are given for the midpoint of the 30 minute exposures. There are 28,30 , and 31 independent radial velocities respectively for M5-8, 12, and 59, while for M5-32 (the only star observed in both 1985 and 1986) we have 56 independent radial velocity measurements. The radial velocity-phase $(\phi)$ curves are plotted for the four stars in Figure 1. The 1986 data are indicated by filled points, while the 1985 data are shown as crosses. The effort to achieve full phase coverage was reasonably successful; only M5-8 has an interval in $\phi$ as large as 0.1 cycles within which there is no accepted radial velocity measurement. Smooth curves were drawn through the $v_{r}-\phi$ curve for each star, and the mean velocities $\left(v_{\gamma}\right)$ were determined. These are 48.0, 53.1, 51.2, and $49.4 \mathrm{~km} \mathrm{~s}^{-1}$, respectively, for the RR Lyrae variables M5-8, 12, 32, and 59. Several different hand-drawn curves which might seem to fit the points for a given star acceptably were tried; the resulting change in $v_{\gamma}$ was always less than $1.2 \mathrm{~km} \mathrm{~s}^{-1}$. Note that the radial velocity for M5 compiled by Webbink (1981) is $51.9 \mathrm{~km} \mathrm{~s}^{-1}$.

\section{b) Photometric Data}

Ideally one desires spectrophotometry covering the entire optical spectral bandpass of the RR Lyraes in M5 which extends over the complete cycle. Although several nights of such data were obtained with the 60 inch $(1.5 \mathrm{~m})$ telescope at Palomar Mountain, using a spectrograph with a 6" slit and a CCD detector, the data were never used because crowding problems in the globular cluster fields prevent one from achieving the necessary accuracy. Instead we resorted to photometry as measured from direct CCD images through wide-band filters. In order to get the maximum possible leverage on temperature variations over the cycle, the filters chosen were the Johnson $B$ filter and a near-infrared $i$ filter adopted for use with CCDs which reproduces the system of Wade et al. (1979) (see also Schneider, Gunn, and Hoessel 1983; Thuan and Gunn 1976). The direct imaging CCD system at the Palomar 60 inch was used to obtain photometry during the five nights of 1986 May 28 through June 1 . The first three of these nights were photometric; the last two were quite cloudy at times. The seeing varied between $1^{\prime \prime}$ and $2^{\prime \prime}$ over the five nights, except when it was extremely cloudy.

An RCA $320 \times 512$ CCD was used mounted at the Cassegrain focus of the 60 inch telescope. The scale was 0 ."46 per pixel. This meant that the four variables for which radial velocity data had been obtained could not be covered in a single exposure, and two fields were necessary. Within the first field, centered near M5-8 and M5-32, were also located the RR Lyrae stars M5-18, 19, 28, and 31. The second field was centered near M5-12 and M5-59, and also included the RR Lyrae M5-79. The exposures were unguided and were 2 minutes through the $B$ filter and 30 seconds through the $i$ filter when it was clear. Frames through each of the two filters were obtained for the first field; then the second field was observed. Except for interruptions due to standard stars and an attempt to confirm a possible supernova, this continued as long as M5 was higher than 2.0 airmasses. Because not all the nights were photometric, local standards were set up within each frame and used to calibrate the data from the last two nights of poor and variable transparency. Occasionally during these two nights, the transparency was so poor that no observing was possible, or when the transparency improved somewhat, only $i$ measurements were made. Some of the frames on these two nights were integations up to 6 minutes long in $B$ and $200 \mathrm{~s}$ in $i$.

The standard stars of the Thuan-Gunn (1976) system were observed repeatedly throughout the photometric nights. These stars also have well-determined $B$ magnitudes, and they define the zero point of our magnitude system. For the M5 RR Lyrae stars, an aperture photometry code with apertures of 5 pixels in radius (2".3) was used to determine magnitudes from the flattened CCD frames. For the most crowded stars, M5-79 and M5-59, an aperture 3 pixels in radius (1".4) was used. (The differential crowding correction between an aperture whose radius was 3 pixels versus 5 pixels was up to $0.1 \mathrm{mag}$ for these two stars.) Aperture corrections to large apertures were derived from the most isolated bright stars on each frame. On the photometric nights, the corrections from the small-aperture measurements onto the magnitude scale set by the local standards clearly showed the effects of deteriorating seeing and transparency when M5 was far from culmination.

The raw data on the photometric nights are extremely good, with more than $10^{4}$ detected electrons above the level of the sky over the stellar image of the RR Lyraes at all phases. The accuracy of the photometry from the last two nights is significantly worse. Other factors which may cause an occasional bad magnitude are cosmic-ray hits, although these were eliminated when we noticed them on the image of a variable, and the variable lying superposed on the single bad column in the detector. This last problem is more serious in the $B$ frames than in the $i$ frames, as the brighter sky in the latter tended to fill in the bad column to some extent. The $B$ and $i$ magnitudes, together with the Julian date of the mean time of the $B$ and $i$ frame, are listed in Table 3A for the field including M5-12 and M5-59, and in Table 3B for the second field. They are plotted as a function of phase in Figure 2. There are about 90 pairs of measurements for each variable, except M5-19, which was often close to the edge of the field and sometimes completely off the edge of the frames.

These $B$ and $i$ magnitudes were transformed to $V$ magnitudes using the relationship

$$
V=B-0.519(B-i)-0.293
$$


TABLE 2

Radial Velocities of M5 RR LyRae Stars

\begin{tabular}{|c|c|c|c|c|c|c|c|c|c|c|c|}
\hline \multicolumn{3}{|c|}{ M5-8 } & \multicolumn{3}{|c|}{ M5-12 } & \multicolumn{3}{|c|}{ M5-32 } & \multicolumn{3}{|c|}{ M5-59 } \\
\hline $\begin{array}{l}\text { Julian Date } \\
-2446000\end{array}$ & Phase & $\begin{array}{c}\mathrm{V}_{r} \\
\left(\mathrm{kms}^{-1}\right)\end{array}$ & $\begin{array}{c}\text { Julian Date } \\
-2446000\end{array}$ & Phase & $\begin{array}{c}\mathrm{V}_{r} \\
\left(\mathrm{kms}^{-1}\right)\end{array}$ & $\begin{array}{c}\text { Julian Date } \\
-2446000\end{array}$ & Phase & $\begin{array}{c}\mathrm{V}_{r} \\
\left(\mathrm{~km} \mathrm{~s}^{-1}\right)\end{array}$ & $\begin{array}{c}\text { Julian Date } \\
-2446000\end{array}$ & Phase & $\begin{array}{c}\mathrm{V}_{r} \\
\left(\mathrm{~km} \mathrm{~s}^{-1}\right)\end{array}$ \\
\hline 538.898 & 0.381 & 49.4 & 565.653 & 0.334 & 44.0 & 184.749 & 0.436 & 53.1 & 565.653 & 0.249 & 31.5 \\
\hline 538.922 & 0.424 & 50.3 & 565.677 & 0.384 & 53.8 & 184.771 & 0.483 & 60.6 & 565.677 & 0.293 & 39.5 \\
\hline 538.945 & 0.467 & 55.3 & 565.699 & 0.432 & 51.5 & 184.799 & 0.544 & 64.7 & 565.699 & 0.334 & 41.7 \\
\hline 538.992 & 0.554 & 59.2 & 565.720 & 0.476 & 56.0 & 184.823 & 0.597 & 60.2 & 565.720 & 0.372 & 45.4 \\
\hline 539.739 & 0.921 & 47.1 & 566.674 & 0.515 & 66.0 & 184.845 & 0.645 & 72.3 & 566.694 & 0.170 & 30.2 \\
\hline 539.763 & 0.965 & 23.3 & 566.697 & 0.564 & 73.6 & 184.867 & 0.693 & 78.3 & 566.697 & 0.174 & 33.5 \\
\hline 539.786 & 0.007 & 18.4 & 566.719 & 0.613 & 77.8 & 186.740 & 0.784 & 71.2 & 566.719 & 0.216 & 38.1 \\
\hline 539.810 & 0.052 & 21.5 & 566.742 & 0.662 & 76.8 & 186.762 & 0.832 & 71.5 & 566.742 & 0.258 & 41.4 \\
\hline 539.834 & 0.095 & 20.7 & 566.765 & 0.709 & 81.2 & 186.785 & 0.882 & 72.9 & 566.765 & 0.299 & 42.6 \\
\hline 539.857 & 0.137 & 27.8 & 566.788 & 0.758 & 77.9 & 186.807 & 0.931 & 81.1 & 566.788 & 0.342 & 47.3 \\
\hline 539.880 & 0.179 & 22.2 & 566.810 & 0.806 & 76.2 & 186.828 & 0.978 & 25.6 & 566.810 & 0.383 & 48.5 \\
\hline 539.903 & 0.222 & 29.1 & 566.891 & 0.980 & 24.0 & 186.851 & 0.026 & 10.1 & 566.891 & 0.532 & 63.4 \\
\hline 565.758 & 0.555 & 58.1 & 566.940 & 0.085 & 25.0 & 186.875 & 0.080 & 14.6 & 566.940 & 0.623 & 66.1 \\
\hline 565.784 & 0.602 & 68.4 & 567.665 & 0.634 & 77.9 & 186.897 & 0.127 & 24.9 & 567.665 & 0.960 & 30.5 \\
\hline 565.806 & 0.643 & 71.4 & 567.687 & 0.681 & 79.1 & 186.919 & 0.177 & 29.9 & 567.687 & 0.001 & 26.0 \\
\hline 565.829 & 0.685 & 65.6 & 567.709 & 0.729 & 82.9 & 186.942 & 0.225 & 35.2 & 567.709 & 0.042 & 28.0 \\
\hline 565.864 & 0.748 & 67.5 & 567.731 & 0.776 & 80.6 & 186.986 & 0.322 & 50.6 & 567.731 & 0.083 & 29.3 \\
\hline 565.879 & 0.776 & 70.7 & 567.754 & 0.825 & 80.6 & 187.697 & 0.874 & 71.4 & 567.754 & 0.125 & 33.0 \\
\hline 566.845 & 0.545 & 68.7 & 567.776 & 0.873 & 83.6 & 187.722 & 0.929 & 57.9 & 567.776 & 0.166 & 33.2 \\
\hline 566.874 & 0.597 & 67.4 & 567.826 & 0.978 & 26.4 & 187.751 & 0.994 & 45.2 & 567.826 & 0.257 & 47.3 \\
\hline 566.926 & 0.692 & 63.1 & 567.848 & 0.026 & 23.2 & 187.776 & 0.047 & 12.9 & 567.838 & 0.298 & 51.2 \\
\hline 567.812 & 0.315 & 45.6 & 567.901 & 0.140 & 31.8 & 187.808 & 0.117 & 19.1 & 567.901 & 0.397 & 48.0 \\
\hline 567.888 & 0.453 & 54.0 & 568.635 & 0.708 & 74.7 & 187.831 & 0.168 & 28.0 & 567.987 & 0.555 & 55.3 \\
\hline 568.693 & 0.928 & 35.0 & 568.707 & 0.862 & 78.3 & 187.855 & 0.220 & 30.7 & 568.635 & 0.750 & 68.5 \\
\hline 568.742 & 0.017 & 21.0 & 568.755 & 0.965 & 22.8 & 187.878 & 0.272 & 33.5 & 568.707 & 0.883 & 70.2 \\
\hline 568.790 & 0.105 & 20.4 & 568.855 & 0.179 & 34.4 & 187.908 & 0.337 & 45.8 & 568.755 & 0.971 & 24.5 \\
\hline 568.812 & 0.145 & 21.2 & 568.877 & 0.226 & 38.8 & 538.898 & 0.047 & 17.8 & 568.855 & 0.156 & 28.0 \\
\hline \multirow[t]{29}{*}{568.834} & 0.186 & 26.1 & 568.898 & 0.271 & 42.0 & 538.922 & 0.099 & 15.8 & 568.877 & 0.197 & 34.8 \\
\hline & & & 568.919 & 0.317 & 44.9 & 538.945 & 0.150 & 17.9 & & & \\
\hline & & & 568.940 & 0.361 & 49.8 & 538.968 & 0.200 & 22.5 & & & \\
\hline & & & & & & 538.992 & 0.253 & 22.7 & & & \\
\hline & & & & & & 539.739 & 0.884 & 53.3 & & & \\
\hline & & & & & & 539.763 & 0.937 & 40.0 & & & \\
\hline & & & & & & 539.786 & 0.987 & 15.2 & & & \\
\hline & & & & & & 539.810 & 0.040 & 18.9 & & & \\
\hline & & & & & & 539.834 & 0.092 & 15.7 & & & \\
\hline & & & & & & 539.857 & 0.142 & 16.9 & & & \\
\hline & & & & & & 539.880 & 0.192 & 24.2 & & & \\
\hline & & & & & & 539.903 & 0.243 & 26.2 & & & \\
\hline & & & & & & 539.937 & 0.316 & 35.8 & & & \\
\hline & & & & & & 565.758 & 0.721 & 58.6 & & & \\
\hline & & & & & & 565.784 & 0.778 & 67.8 & & & \\
\hline & & & & & & 565.829 & 0.876 & 64.6 & & & \\
\hline & & & & & & 565.864 & 0.952 & 62.7 & & & \\
\hline & & & & & & 565.879 & 0.985 & 22.7 & & & \\
\hline & & & & & & 565.925 & 0.086 & 7.5 & & & \\
\hline & & & & & & 566.845 & 0.096 & 20.7 & & & \\
\hline & & & & & & 566.874 & 0.158 & 26.3 & & & \\
\hline & & & & & & 566.926 & 0.271 & 32.6 & & & \\
\hline & & & & & & 567.812 & 0.207 & 34.4 & & & \\
\hline & & & & & & 567.888 & 0.372 & 48.0 & & & \\
\hline & & & & & & 568.693 & 0.132 & 25.8 & & & \\
\hline & & & & & & 568.742 & 0.238 & 34.6 & & & \\
\hline & & & & & & 568.790 & 0.343 & 41.5 & & & \\
\hline & & & & & & 568.812 & 0.392 & 47.7 & & & \\
\hline & & & & & & 568.834 & 0.440 & 51.1 & & & \\
\hline
\end{tabular}




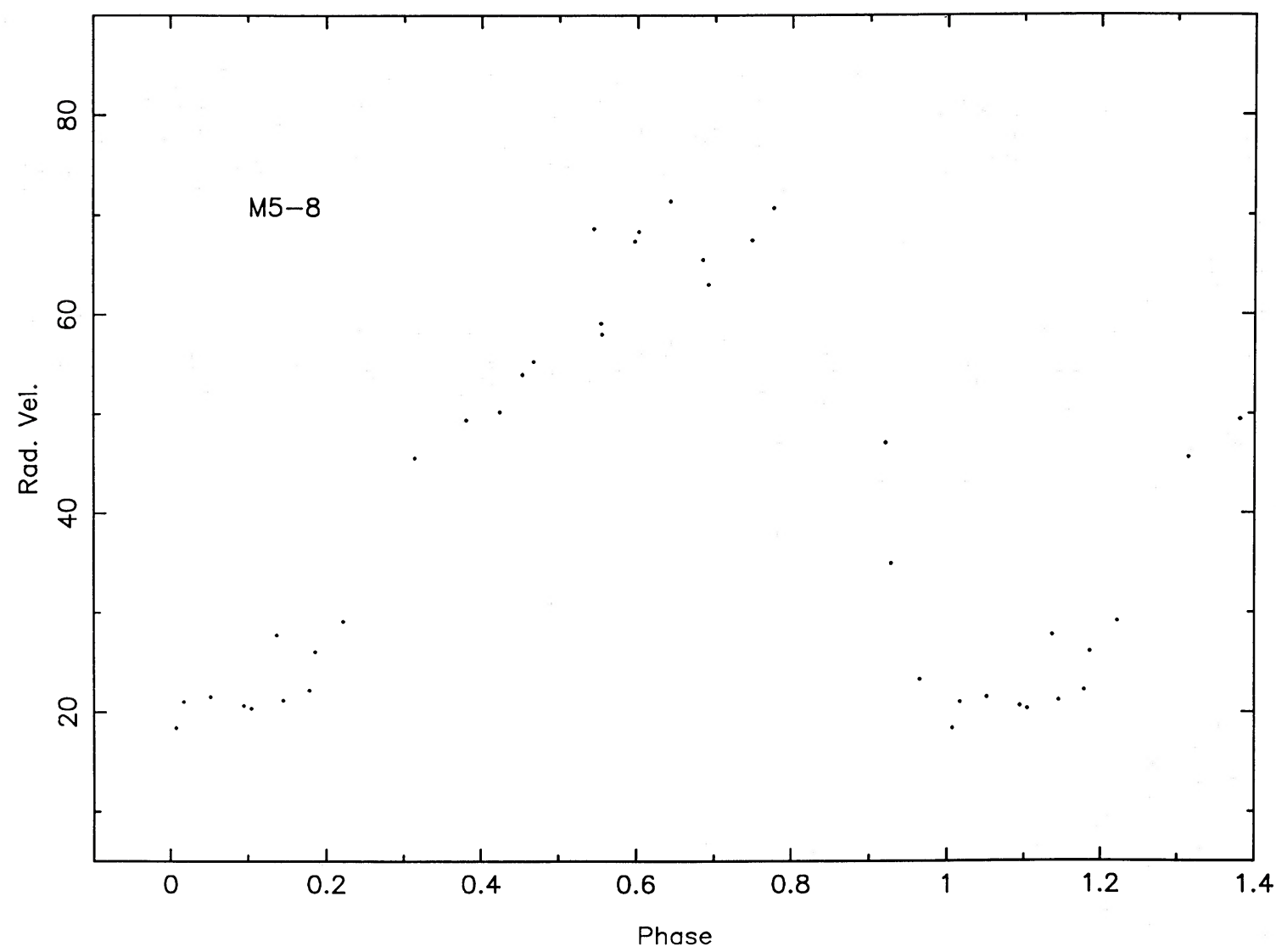

FIG. $1 a$

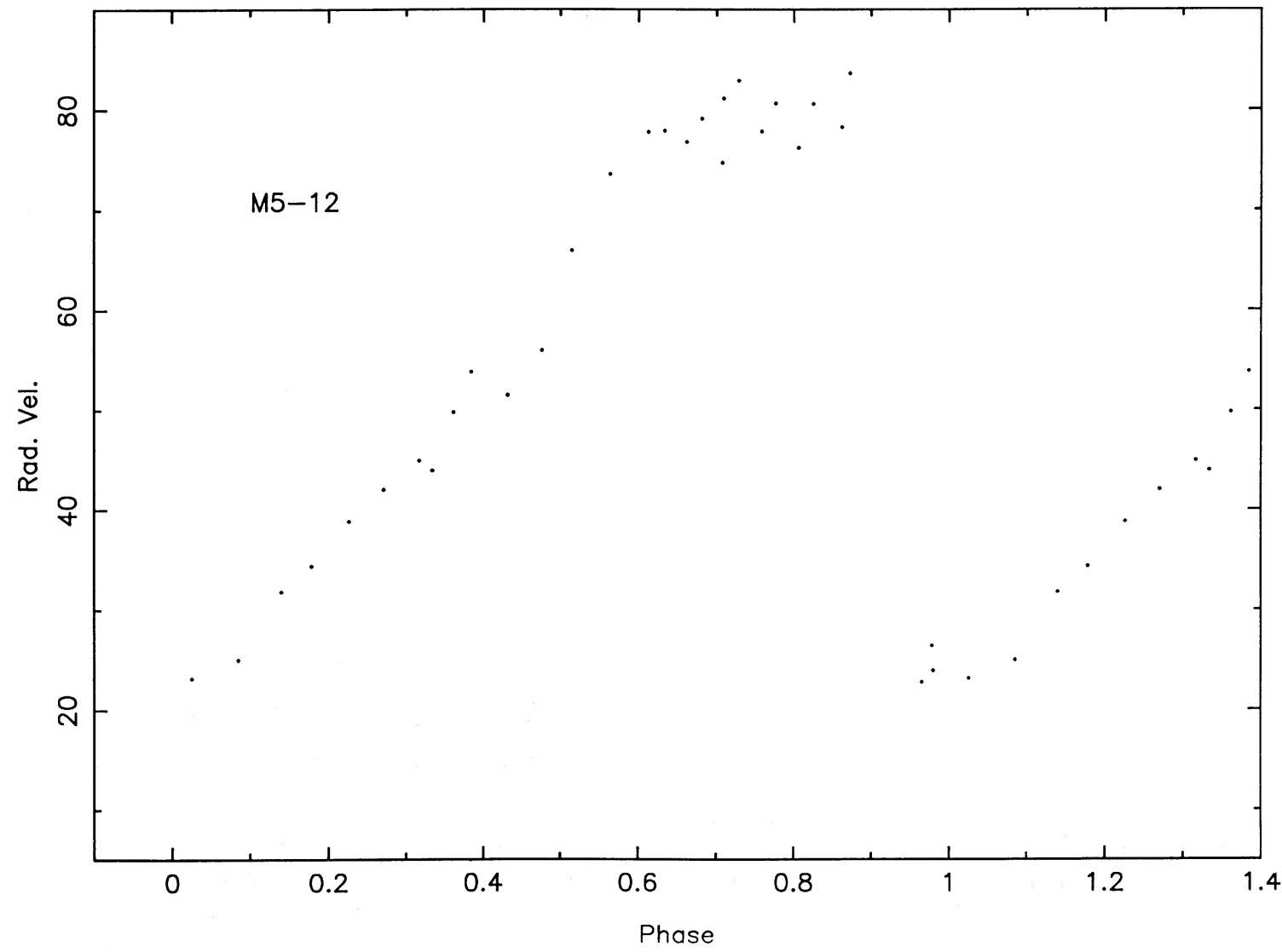

FIG. $1 b$

FIG. 1.-The radial velocity for the RR Lyrae stars M5-8, M5-12, M5-32, and M5-59 is shown as a function of phase. The points represent the 1986 measurements, while the 1985 measurements for M5-32 are shown by crosses. 


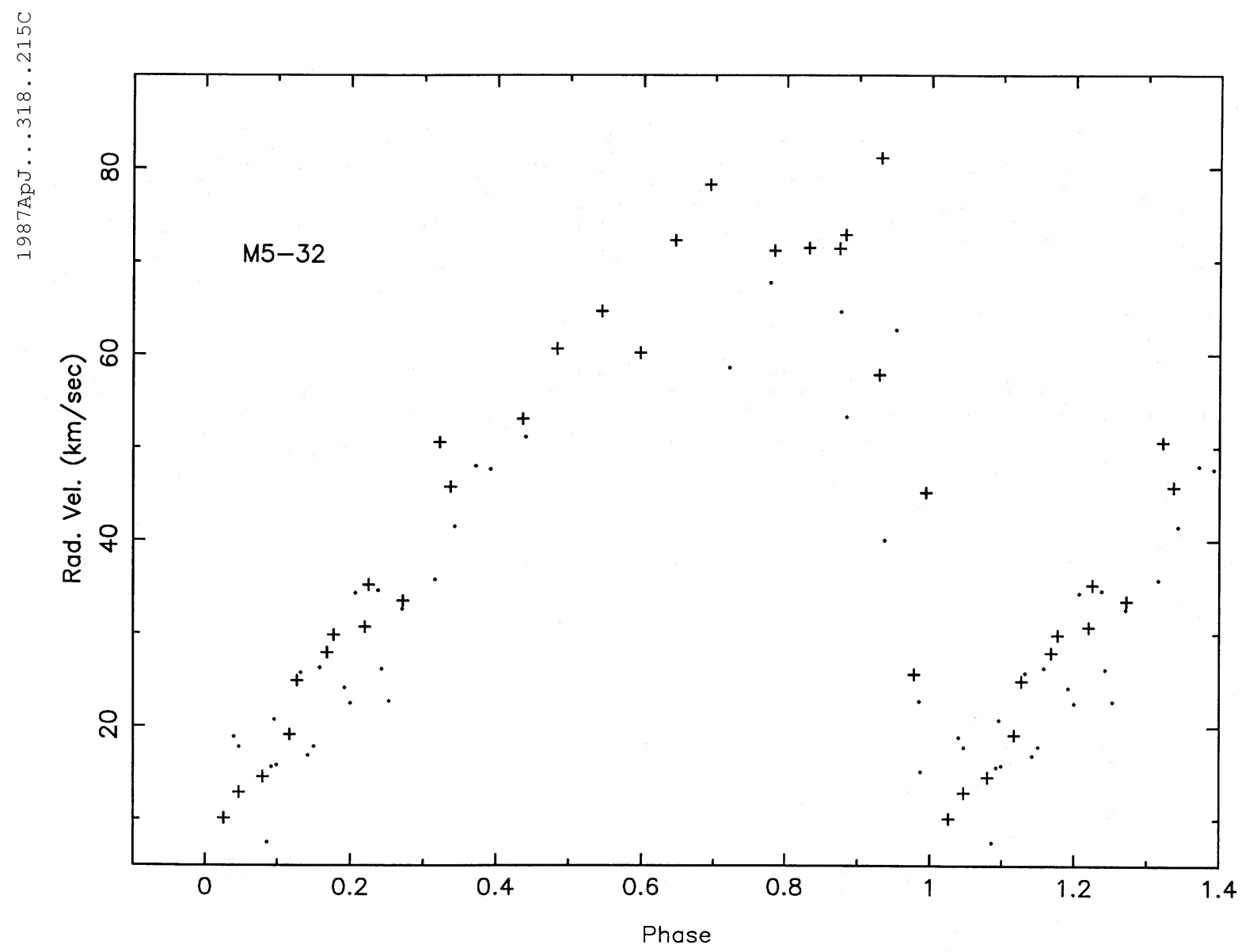

FIG. 1c

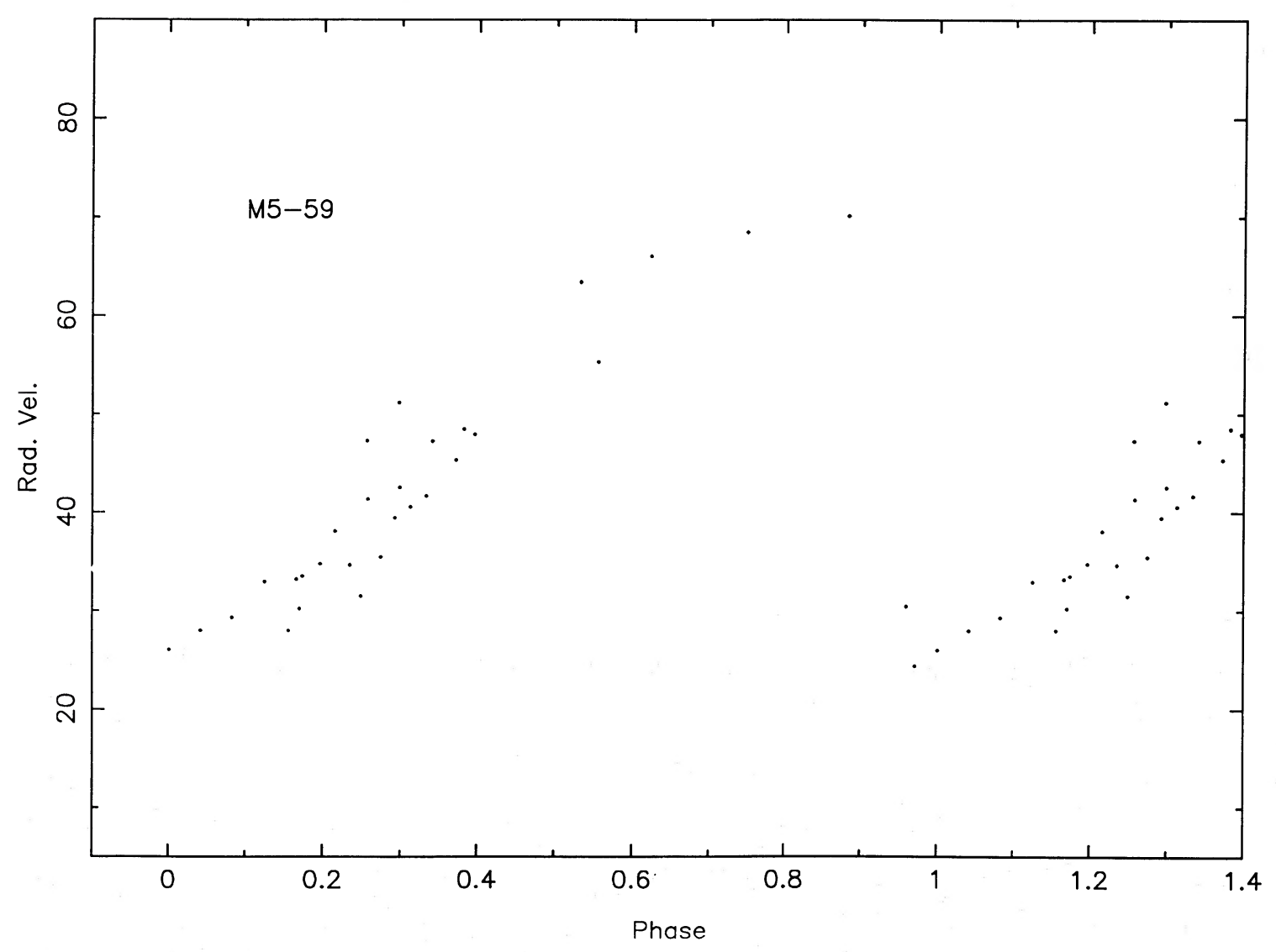

FIG $1 d$ 
TABLE 3A

B AND $i$ PhOTOMETRY-M5 FiELd 1

\begin{tabular}{|c|c|c|c|c|c|c|c|c|c|c|c|c|c|}
\hline \multirow[b]{2}{*}{$\begin{array}{c}\text { Julian Day } \\
-2446000\end{array}$} & \multicolumn{2}{|c|}{ M5-59 } & \multicolumn{2}{|c|}{ M5-12 } & \multicolumn{2}{|c|}{ M5-79 } & \multirow[b]{2}{*}{$\begin{array}{c}\text { Julian Day } \\
-2446000\end{array}$} & \multicolumn{2}{|c|}{ M5-59 } & \multicolumn{2}{|c|}{ M5-12 } & \multicolumn{2}{|c|}{ M5-79 } \\
\hline & $\begin{array}{c}\mathrm{B} \\
(\mathrm{mag}) \\
\end{array}$ & $\stackrel{\mathrm{i}}{(\mathrm{mag})}$ & $\begin{array}{c}\mathrm{B} \\
(\mathrm{mag})\end{array}$ & $\begin{array}{c}\mathrm{i} \\
(\mathrm{mag})\end{array}$ & $\begin{array}{c}\mathrm{B} \\
(\mathrm{mag})\end{array}$ & $\stackrel{i}{\mathrm{i}}$ & & $\begin{array}{c}\mathrm{B} \\
(\mathrm{mag})\end{array}$ & $\begin{array}{c}\mathrm{i} \\
(\mathrm{mag})\end{array}$ & $\begin{array}{c}\mathrm{B} \\
(\mathrm{mag})\end{array}$ & $\stackrel{\mathrm{i}}{(\mathrm{mag})}$ & $\begin{array}{c}\text { B } \\
(\mathrm{mag})\end{array}$ & $\begin{array}{c}\mathrm{i} \\
(\mathrm{mag})\end{array}$ \\
\hline 578.669 & 15.349 & 15.022 & 15.081 & 15.145 & 15.546 & 15.250 & 580.680 & 14.609 & 14.813 & 15.870 & 15.483 & 15.405 & $\overline{15.186}$ \\
\hline 578.680 & 15.393 & 15.045 & 15.174 & 15.188 & 15.507 & 15.237 & 580.701 & 14.611 & 14.790 & 15.939 & 15.531 & 15.339 & 15.175 \\
\hline 578.688 & 15.414 & 15.067 & 15.219 & 15.199 & 15.453 & 15.255 & 580.711 & 14.668 & 14.814 & 15.972 & 15.549 & 15.264 & 15.161 \\
\hline 578.705 & 15.480 & 15.119 & 15.338 & 15.266 & 15.343 & 15.194 & 580.728 & 14.790 & 14.890 & 15.965 & 15.569 & 15.190 & 15.130 \\
\hline 578.717 & 15.531 & 15.101 & 15.415 & 15.292 & 15.249 & 15.176 & 580.742 & 14.855 & 14.901 & 15.972 & 15.580 & 15.122 & 15.084 \\
\hline 578.724 & 15.548 & 15.127 & 15.441 & 15.308 & 15.213 & 15.137 & 580.754 & 14.926 & 14.917 & 15.969 & 15.585 & 15.115 & 15.119 \\
\hline 578.733 & 15.562 & 15.107 & 15.501 & 15.331 & 15.149 & 15.088 & 580.766 & 15.000 & 14.962 & 15.943 & 15.616 & 15.057 & 15.087 \\
\hline 578.741 & 15.591 & 15.142 & 15.543 & 15.326 & 15.153 & 15.087 & 580.776 & 15.046 & 14.964 & 15.959 & 15.575 & 15.060 & 15.042 \\
\hline 578.753 & 15.623 & 15.157 & 15.543 & 15.375 & 15.095 & 15.062 & 580.788 & 15.120 & 15.008 & 15.926 & 15.615 & 15.041 & 15.045 \\
\hline 578.778 & 15.653 & 15.168 & 15.717 & 15.455 & 15.070 & 15.049 & 580.806 & 15.211 & 15.009 & 15.958 & 15.574 & 15.071 & 15.061 \\
\hline 578.785 & 15.687 & 15.175 & 15.749 & 15.434 & 15.062 & 15.024 & 580.820 & 15.247 & 15.016 & 15.957 & 15.605 & 15.090 & 15.069 \\
\hline 578.797 & 15.695 & 15.197 & 15.807 & 15.451 & 15.062 & 15.042 & 580.829 & 15.294 & 15.012 & 15.907 & 15.653 & 15.115 & 15.035 \\
\hline 578.804 & 15.708 & 15.191 & 15.829 & 15.457 & 15.050 & 15.052 & 580.840 & 15.344 & 15.057 & 15.930 & 15.591 & 15.176 & 15.083 \\
\hline 578.820 & 15.716 & 15.229 & 15.902 & 15.488 & 15.106 & 15.055 & 580.855 & 15.367 & 15.057 & 15.973 & 15.629 & 15.203 & 15.105 \\
\hline 578.828 & 15.713 & 15.199 & 15.920 & 15.503 & 15.125 & 15.064 & 581.668 & 15.808 & 15.332 & 16.007 & 15.531 & 15.422 & 15.225 \\
\hline 578.844 & 15.727 & 15.219 & 15.956 & 15.537 & 15.199 & 15.108 & 581.690 & 15.859 & 15.336 & 16.004 & 15.659 & 15.361 & 15.163 \\
\hline 578.854 & 15.724 & 15.217 & 15.975 & 15.548 & 15.231 & 15.108 & 581.726 & 15.635 & 15.337 & 15.922 & 15.576 & 15.141 & 15.078 \\
\hline 578.868 & 15.719 & 15.265 & 15.946 & 15.542 & 15.323 & 15.150 & 581.747 & 15.058 & 15.060 & 15.942 & 15.620 & 15.094 & 15.086 \\
\hline 578.880 & 15.712 & 15.243 & 15.931 & 15.547 & 15.339 & 15.214 & 581.764 & 14.707 & 14.852 & 15.883 & 15.507 & 15.116 & 15.078 \\
\hline 578.890 & 15.759 & 15.225 & 15.932 & 15.604 & 15.405 & 15.206 & 581.781 & 14.618 & 14.814 & 15.959 & 15.634 & 15.116 & 15.095 \\
\hline 578.900 & 15.744 & 15.243 & 15.936 & 15.579 & 15.438 & 15.184 & 581.803 & 14.743 & 14.842 & 16.027 & 15.709 & 15.088 & 15.057 \\
\hline 578.912 & 15.750 & 15.243 & 15.971 & 15.573 & 15.499 & 15.222 & 581.818 & 14.825 & 14.878 & 15.957 & 15.690 & 15.075 & 15.053 \\
\hline 578.920 & 15.735 & 15.293 & 15.936 & 15.569 & 15.519 & 15.217 & 581.833 & 14.923 & 14.889 & 15.624 & 15.540 & 15.145 & 15.053 \\
\hline 579.653 & 14.849 & 14.860 & 15.414 & 15.325 & 15.531 & 15.217 & 581.851 & 15.011 & 14.954 & 14.653 & 15.073 & 15.182 & 15.074 \\
\hline 579.663 & 14.917 & 14.897 & 15.493 & 15.291 & 15.525 & 15.266 & 581.860 & 15.082 & 14.995 & 14.424 & 14.920 & 15.232 & 15.115 \\
\hline 579.670 & 14.947 & 14.936 & 15.506 & 15.311 & 15.506 & 15.240 & 581.871 & 15.115 & 14.975 & 14.408 & 14.904 & 15.327 & 15.159 \\
\hline 579.688 & 15.033 & 14.967 & 15.585 & 15.363 & 15.435 & 15.212 & 581.880 & 15.173 & 15.014 & 14.483 & 14.931 & 15.336 & 15.121 \\
\hline 579.695 & 15.127 & 14.980 & 15.619 & 15.386 & 15.386 & 15.196 & 581.889 & 15.226 & 14.986 & 14.581 & 14.924 & 15.375 & 15.158 \\
\hline 579.714 & 15.169 & 14.982 & 15.697 & 15.407 & 15.253 & 15.146 & 581.907 & 15.261 & 15.037 & 14.724 & 15.020 & 15.447 & 15.217 \\
\hline 579.721 & 15.207 & 14.999 & 15.728 & 15.450 & 15.214 & 15.141 & 581.916 & 15.318 & 15.040 & 14.812 & 15.072 & 15.461 & 15.220 \\
\hline 579.743 & 15.310 & 15.027 & 15.854 & 15.487 & 15.182 & 15.112 & 582.649 & 15.858 & 15.252 & 15.795 & 15.541 & 15.567 & 15.289 \\
\hline 579.752 & 15.332 & 15.047 & 15.871 & 15.481 & 15.139 & 15.097 & 582.661 & 15.741 & 15.230 & 15.905 & 15.596 & 15.512 & 15.246 \\
\hline 579.774 & 15.415 & 15.065 & 15.931 & 15.512 & 15.015 & 15.036 & 582.667 & 15.733 & 15.231 & 15.941 & 15.581 & 15.519 & 15.234 \\
\hline 579.783 & 15.463 & 15.072 & 15.973 & 15.539 & 14.999 & 15.029 & 582.685 & 15.711 & 15.253 & 15.931 & 15.585 & 15.400 & 15.220 \\
\hline 579.799 & 15.491 & 15.083 & 15.968 & 15.516 & 15.022 & 15.034 & 582.697 & 15.787 & 15.312 & 15.968 & 15.527 & 15.317 & 15.138 \\
\hline 579.806 & 15.526 & 15.113 & 15.967 & 15.558 & 15.065 & 15.039 & 582.712 & 15.748 & 15.214 & 15.970 & 15.612 & 15.210 & 15.122 \\
\hline 579.819 & 15.572 & 15.118 & 15.881 & 15.539 & 14.989 & 15.045 & 582.726 & 15.720 & 15.236 & 20.00 & 15.628 & 15.307 & 15.054 \\
\hline 579.830 & 15.605 & 15.136 & 15.962 & 15.573 & 15.154 & 15.047 & 582.769 & & 15.363 & & 15.429 & & 15.072 \\
\hline 579.846 & 15.659 & 15.134 & 15.949 & 15.585 & 15.225 & 15.099 & 582.784 & 15.854 & 15.352 & 14.654 & 15.119 & 15.126 & 15.048 \\
\hline 579.860 & 15.684 & 15.185 & 15.964 & 15.571 & 15.283 & 15.116 & 582.811 & 15.645 & 15.296 & 14.471 & 14.899 & 15.109 & 15.013 \\
\hline 579.874 & 15.687 & 15.178 & 15.957 & 15.574 & 15.339 & 15.140 & 582.831 & 15.120 & 15.024 & 14.645 & 14.979 & & 15.078 \\
\hline 579.896 & 15.724 & 15.207 & 15.940 & 15.591 & 15.425 & 15.188 & 582.839 & 15.005 & 14.961 & 14.725 & 15.026 & 15.159 & 15.089 \\
\hline 579.902 & 15.717 & 15.190 & 15.953 & 15.613 & 15.429 & 15.186 & 582.847 & 14.822 & 14.849 & 14.797 & 15.043 & 15.210 & 15.120 \\
\hline 579.913 & 15.720 & 15.191 & 15.988 & 15.574 & 15.485 & 15.183 & 582.856 & 14.637 & 14.825 & 14.873 & 15.095 & 15.256 & 15.152 \\
\hline 579.920 & 15.729 & 15.221 & 16.050 & 15.684 & 15.508 & 15.230 & 582.862 & 14.584 & 14.792 & 14.921 & 15.079 & 15.258 & 15.128 \\
\hline 580.657 & 15.113 & 15.087 & 15.746 & 15.452 & 15.473 & 15.264 & & & & & & & \\
\hline 580.668 & 15.078 & 14.981 & 15.795 & 15.442 & 15.469 & 15.202 & & & & & & & \\
\hline 580.675 & 14.893 & 14.891 & 15.825 & 15.456 & 15.468 & 15.222 & & & & & & & \\
\hline
\end{tabular}

which was derived from bright standard stars covering a range in $B-i$ color which exceeded $2.7 \mathrm{mag}$. For each variable, the $V$ magnitudes were averaged over the cycle (in bins of 0.02 in $\phi$ ) to determine the mean $V$ color, and were converted to intensities, then averaged over the cycle, then converted back to a magnitude, to yield the intensity mean $V$ magnitude. These parameters, together with the minimum and maximum of the light curves in $B, i$, and $V$, are listed in Table 1 . An interstellar absorption correction at $V$ of $0.093 \mathrm{mag}$ has been adopted for M5 (Zinn 1980).

\section{DERIVATION OF DISTANCES AND MEAN RADII}

\section{a) Method of Analysis}

The analysis of this data has as its goal the derivation of the angular diameter (henceforth $\theta$ ) as a function of phase from both the radial velocity and the photometric data sets. Forcing agreement between these two $\theta(\phi)$ relationships determines the distance to the pulsating variable star. The manipulation of the radial velocities is straightforward. A hand-drawn curve which fits the radial velocity $-\phi$ relationship for each M5 RR Lyrae was digitized at 0.02 cycle intervals. The pulsational velocity is the difference between $v_{r}$ and the mean velocity $v_{\gamma}$, defined in $\S$ II $a$, multiplied by the projection factor $p$ to convert a line-ofsight velocity into a pulsational velocity. This factor has been set at 1.31. Values adopted in recent analyses in the literature range from 1.30 to 1.35 ; the choice of $p$ and the accuracy to which it is known are discussed in Hindsley and Bell (1986). If $p$ is underestimated by $5 \%$, the distance to the pulsating variable star will be underestimated by $5 \%$.

The pulsational velocity is then integrated over time (i.e., phase $\times$ the period of the variable) to yield the difference in radius $\delta R(\phi)$. The integration is straightforward and has been checked by reproducing the results of Oke (1966) from the $v_{r}-\phi$ curve for the field RR Lyrae star X Ari. We begin the integration just past maximum light when the star begins to expand, 
as that puts the worst determined part of the pulsational velocity curve, that of the rapid drop in radial velocity at maximum light, at the end of the integration. If a value for the radius at $\phi=0$ and one for the distance to M5 are assumed, the angular diameter over the cycle is easily obtained.

Ideally we want to measure a radial velocity from a spectral feature arising at the same optical depth where the continuum is formed; this is clearly impossible. We have used the $\mathrm{Mg}$ triplet region, which in these metal-poor stars contains only relatively weak absorption lines. However, one might be concerned, should a strong gradient in pulsational velocity with depth exist, that the varying depth of line formation for a given spectral feature (due to the varying $T_{\text {eff }}$ with phase) as a function of phase could lead to a radial velocity curve which does not follow the pulsational velocity. Fortunately Jones et al. (1987a) have already demonstrated that the velocity gradient with depth, at least over the relevant range of optical depth, is small. We are thus confident that the observed radial velocity curve is a true reflection of the pulsational velocity as a function of phase.

The derivation of the $\theta-\phi$ relationship from the photometric data is more complex, and several different schemes have been applied in the literature. We have developed an independent analysis which has the advantage that the sources of uncertainty are much more clearly visible. We have measured $B-i$ colors as a function of phase. We use the grid of models of Kurucz (1979), adopting $Z / Z_{0}=1 / 10$. (Frogel, Cohen, and Persson 1983 deduced $[\mathrm{Fe} / \mathrm{H}](\mathrm{M} 5)=-1.5 \mathrm{dex}$, while Zinn and West 1984 derived -1.4 dex. The error introduced by not interpolating properly within the Kurucz grid between models of 0.1 and 0.01 solar metallicity is discussed in $\S \mathrm{IV} a$ and is not significant.) These models are used setting the effective wavelength of the $B$ filter as $4512 \AA$ and that of the $i$ filter as $8050 \AA$ to define the dependence of the $B-i$ color on $T_{\text {eff }}$ and surface gravity. The zero point of the $B-i$ colors is fixed by the analysis of Bell and Oke (1986) of the bright metal-poor sub-

TABLE 3B

$B$ AND $i$ PHOTOMETRY-M5 Field 2

\begin{tabular}{|c|c|c|c|c|c|c|c|c|c|c|c|c|}
\hline \multirow[b]{2}{*}{$\begin{array}{l}\text { Julian Day } \\
-2446000\end{array}$} & \multicolumn{2}{|c|}{ M5-32 } & \multicolumn{2}{|c|}{ M5-8 } & \multicolumn{2}{|c|}{ M5-19 } & \multicolumn{2}{|c|}{ M5-31 } & \multicolumn{2}{|c|}{ M5-28 } & \multicolumn{2}{|c|}{ M5-18 } \\
\hline & $\begin{array}{c}\mathrm{B} \\
\text { (mag) }\end{array}$ & $\begin{array}{c}\mathrm{i} \\
(\mathrm{mag})\end{array}$ & $\begin{array}{c}\text { B } \\
\text { (mag) }\end{array}$ & $\begin{array}{c}\mathrm{i} \\
(\mathrm{mag})\end{array}$ & $\underset{(\mathrm{mag})}{\mathrm{B}}$ & $\begin{array}{c}\mathrm{i} \\
(\mathrm{mag})\end{array}$ & $\begin{array}{c}\mathrm{B} \\
(\mathrm{mag})\end{array}$ & $\begin{array}{c}\mathrm{i} \\
(\mathrm{mag})\end{array}$ & $\begin{array}{c}\text { B } \\
\text { (mag) }\end{array}$ & $\begin{array}{c}\mathrm{i} \\
\text { (mag) }\end{array}$ & $\begin{array}{c}\mathrm{B} \\
(\mathrm{mag})\end{array}$ & $\begin{array}{c}\mathrm{i} \\
(\mathrm{mag})\end{array}$ \\
\hline 578.675 & 15.599 & 15.398 & 15.339 & 15.232 & & & 15.681 & 15.547 & 15.395 & 15.210 & 15.052 & 15.156 \\
\hline 578.685 & 15.044 & 15.140 & 15.367 & 15.213 & & & 15.674 & 15.580 & 15.446 & 15.199 & 15.131 & 15.162 \\
\hline 578.691 & 14.674 & 14.951 & 15.420 & 15.265 & & & 15.679 & 15.575 & 15.487 & 15.241 & 15.166 & 15.179 \\
\hline 578.710 & 14.419 & 14.868 & 15.454 & 15.233 & & & 15.667 & 15.576 & 15.533 & 15.215 & 15.311 & 15.223 \\
\hline 578.721 & 14.456 & 14.896 & 15.491 & 15.231 & & & 15.659 & 15.575 & 15.552 & 15.251 & 15.366 & 15.243 \\
\hline 578.737 & 14.626 & 14.978 & 15.555 & 15.275 & & & 15.609 & 15.527 & 15.603 & 15.278 & 15.464 & 15.284 \\
\hline 578.750 & 14.745 & 15.036 & 15.597 & 15.268 & & & 15.465 & 15.471 & 15.630 & 15.255 & 15.524 & 15.309 \\
\hline 578.758 & 14.824 & 15.065 & 15.625 & 15.278 & & & 15.338 & 15.404 & 15.649 & 15.277 & 15.555 & 15.333 \\
\hline 578.779 & 15.057 & 15.124 & 15.710 & 15.333 & & & 15.059 & 15.289 & 15.744 & 15.288 & 15.657 & 15.355 \\
\hline 578.789 & 15.131 & 15.176 & 15.734 & 15.327 & 16.068 & 15.626 & 15.098 & 15.324 & 15.778 & 15.329 & 15.675 & 15.330 \\
\hline 578.801 & 15.211 & 15.227 & 15.777 & 15.338 & 16.044 & 15.644 & 15.065 & 15.256 & 15.773 & 15.341 & 15.716 & 15.403 \\
\hline 578.808 & 15.259 & 15.249 & 15.784 & 15.338 & 15.908 & & 15.055 & 15.290 & & 15.313 & 15.722 & 15.420 \\
\hline 578.824 & 15.390 & 15.257 & 15.862 & 15.368 & & 15.636 & 15.022 & 15.240 & 15.873 & 15.367 & 15.748 & 15.413 \\
\hline 578.833 & 15.401 & 15.295 & 15.841 & 15.391 & 16.028 & 15.643 & 15.028 & 15.279 & 15.827 & 15.373 & 15.747 & 15.417 \\
\hline 578.848 & 15.523 & 15.332 & 15.850 & 15.427 & & 15.645 & 15.088 & 15.277 & & 15.360 & 15.770 & 15.442 \\
\hline 578.858 & 15.541 & 15.366 & 15.889 & 15.410 & 15.968 & & 15.102 & 15.286 & 15.844 & 15.368 & 15.752 & 15.42 ? \\
\hline 578.872 & 15.629 & 15.401 & 15.897 & 15.454 & 15.970 & 15.627 & 15.192 & 15.432 & 15.871 & 15.431 & 15.786 & 15.487 \\
\hline 578.883 & 15.735 & 15.407 & 15.946 & 15.455 & 16.022 & 15.621 & 15.315 & 15.425 & 15.898 & 15.416 & 15.872 & 15.459 \\
\hline 578.892 & 15.718 & 15.439 & 15.909 & 15.449 & 15.975 & 15.660 & 15.303 & 15.487 & 15.844 & 15.419 & 15.825 & 15.493 \\
\hline 578.906 & 15.814 & 15.447 & 15.883 & 15.424 & 15.993 & 15.684 & 15.425 & 15.483 & 15.883 & 15.428 & 15.820 & 15.482 \\
\hline 578.915 & 15.822 & 15.482 & 15.893 & 15.458 & 15.869 & 15.617 & 15.437 & 15.549 & 15.839 & 15.408 & 15.799 & 15.462 \\
\hline 578.924 & 15.852 & 15.485 & 15.897 & 15.479 & 15.621 & 15.513 & 15.454 & 15.450 & 15.854 & 15.505 & 15.784 & 15.465 \\
\hline 579.660 & 14.698 & 15.024 & 14.670 & 14.913 & 15.921 & 15.557 & 15.381 & 15.442 & 14.817 & 14.973 & 15.410 & 15.297 \\
\hline 579.667 & 14.790 & 15.064 & 14.705 & 14.939 & 15.934 & 15.540 & 15.253 & 15.349 & 14.858 & 15.013 & 15.483 & 15.399 \\
\hline 579.675 & 14.850 & 15.090 & 14.749 & 14.964 & 15.964 & 15.534 & 15.152 & 15.303 & 14.920 & 15.016 & 15.560 & 15.401 \\
\hline 579.692 & 15.005 & 15.149 & 14.852 & 15.016 & 15.893 & 15.609 & 15.105 & 15.342 & 15.002 & 15.035 & 15.567 & 15.310 \\
\hline 579.698 & 15.080 & 15.159 & 14.892 & 15.014 & 16.019 & 15.580 & 15.111 & 15.306 & 15.053 & 15.046 & 15.608 & 15.371 \\
\hline 579.717 & 15.224 & 15.246 & 15.016 & 15.063 & 16.044 & 15.624 & 15.028 & 15.254 & 15.146 & 15.098 & 15.681 & 15.37 .4 \\
\hline 579.725 & 15.270 & 15.264 & 15.053 & 15.082 & 16.047 & 15.624 & 15.008 & 15.226 & 15.205 & 15.124 & 15.700 & 15.396 \\
\hline 579.747 & 15.412 & 15.303 & 15.193 & 15.113 & 16.034 & 15.614 & 15.067 & 15.273 & 15.290 & 15.141 & 15.736 & 15.423 \\
\hline 579.756 & 15.464 & 15.337 & 15.235 & 15.130 & 16.043 & 15.6 & 15.099 & 15.298 & 15.343 & 15.142 & 15.728 & $15.41 \mathrm{f}$ \\
\hline 579.778 & 15.585 & 15.360 & 15.328 & 15.160 & 16.028 & 15.644 & 15.181 & 15.329 & 15.454 & 15.194 & 15.729 & $15.266 j$ \\
\hline 579.788 & 15.622 & 15.372 & 15.382 & 15.198 & 16.011 & 15.635 & 15.250 & 15.352 & 15.487 & 15.217 & 15.565 & 15.449 \\
\hline 579.803 & 15.687 & .418 & 15.448 & 15.216 & 15.967 & 15.623 & 15.350 & 15.439 & 15.537 & 15.227 & 15.776 & $15.36 \%$ \\
\hline 579.810 & 15.723 & 15.446 & 15.486 & 15.191 & 15.957 & 15.630 & 15.295 & 15.497 & 15.552 & 15.239 & 15.782 & 15.496 \\
\hline 579.824 & 15.798 & 15.476 & 15.550 & 15.245 & 15.977 & 15.651 & 15.430 & 15.450 & 15.623 & 15.280 & 15.836 & $15.49: 3$ \\
\hline 579.835 & 15.844 & 15.499 & 15.577 & 15.257 & 15.998 & 15.653 & 15.511 & 15.488 & 15.647 & 15.282 & 15.847 & 15.506 \\
\hline 579.850 & 15.880 & 15.522 & 15.647 & 15.331 & 15.912 & 15.578 & 15.584 & 15.480 & 15.702 & 15.292 & 15.816 & 15.603 \\
\hline 579.865 & 15.951 & 15.545 & 15.679 & 15.298 & 15.622 & 15.434 & 15.649 & 15.536 & 15.742 & 15.315 & 15.879 & 15.59 .4 \\
\hline 579.878 & 15.984 & 15.553 & 15.730 & 15.332 & 14.907 & 15.091 & 15.684 & 15.558 & 15.785 & 15.349 & 15.873 & $15.53: 3$ \\
\hline 579.899 & 15.965 & 15.551 & 15.779 & 15.347 & 14.396 & 14.838 & 15.675 & 15.563 & 15.833 & 15.348 & 15.877 & 15.586 \\
\hline 579.906 & 15.947 & 15.554 & 15.795 & 15.344 & 14.418 & 14.872 & 15.703 & 15.558 & 15.821 & 15.379 & 15.861 & 15.611 \\
\hline 579.917 & 15.935 & 15.571 & 15.813 & 15.364 & 14.496 & 14.926 & 15.718 & 15.671 & 15.843 & 15.368 & 15.843 & 15.557 \\
\hline 580.664 & 15.424 & 15.289 & 16.030 & 15.562 & 16.044 & 15.626 & 15.109 & 15.284 & 15.955 & 15.536 & 15.736 & $15.43 !$ \\
\hline 580.671 & 15.430 & 15.289 & 16.015 & 15.570 & 16.022 & 15.641 & 15.148 & 15.293 & 15.850 & 15.491 & 15.836 & 15.571 \\
\hline 580.679 & 15.484 & 15.271 & 16.013 & 15.604 & 16.021 & 15.659 & 15.203 & 15.296 & 15.695 & 15.455 & 15.822 & 15.498 \\
\hline 580.691 & 15.555 & 15.329 & 15.925 & 15.498 & 16.020 & 15.587 & 15.274 & 15.482 & 15.282 & 15.134 & 15.759 & 15.429 \\
\hline 580.696 & 15.593 & 15.349 & 15.879 & 15.523 & 16.017 & 15.606 & 15.306 & 15.467 & 15.170 & 15.153 & 15.773 & $15.44 !$ \\
\hline
\end{tabular}


TABLE 3B-Continued

\begin{tabular}{|c|c|c|c|c|c|c|c|c|c|c|c|c|}
\hline \multirow[b]{2}{*}{$\begin{array}{l}\text { Julian Day } \\
-2446000\end{array}$} & \multicolumn{2}{|c|}{ M5-32 } & \multicolumn{2}{|c|}{ M5-8 } & \multicolumn{2}{|c|}{ M5-19 } & \multicolumn{2}{|c|}{ M5-31 } & \multicolumn{2}{|c|}{ M5-28 } & \multicolumn{2}{|c|}{ M5-18 } \\
\hline & $\begin{array}{c}\mathrm{B} \\
(\mathrm{mag})\end{array}$ & $\underset{(\mathrm{mag})}{\mathrm{i}}$ & $\underset{(\mathrm{mag})}{\mathrm{B}}$ & $\underset{(\mathrm{mag})}{\mathrm{i}}$ & $\underset{(\mathrm{mag})}{\mathrm{B}}$ & $\begin{array}{c}\mathrm{i} \\
(\mathrm{mag})\end{array}$ & $\begin{array}{c}\mathrm{B} \\
(\mathrm{mag})\end{array}$ & $\begin{array}{c}\mathrm{i} \\
(\mathrm{mag})\end{array}$ & $\begin{array}{c}\mathrm{B} \\
(\mathrm{mag})\end{array}$ & $\underset{(\mathrm{mag})}{\mathrm{i}}$ & $\begin{array}{c}\mathrm{B} \\
(\mathrm{mag})\end{array}$ & $\begin{array}{c}\mathrm{i} \\
(\mathrm{mag})\end{array}$ \\
\hline 580.716 & 15.780 & 15.458 & 15.395 & 15.240 & 15.964 & & 15.383 & 15.444 & 14.801 & 14.952 & 15.759 & 15.426 \\
\hline 580.724 & 15.705 & 15.404 & 15.130 & 15.155 & 16.036 & 15.648 & 15.446 & 15.542 & 14.716 & 14.917 & 15.883 & 15.440 \\
\hline 580.739 & 15.763 & 15.447 & 14.787 & 14.995 & 15.975 & 15.632 & 15.540 & 15.481 & 14.765 & 14.939 & 15.875 & 15.637 \\
\hline 580.750 & 15.806 & 15.497 & 14.679 & 14.942 & 15.941 & 15.626 & 15.597 & 15.522 & 14.840 & 14.964 & 15.916 & 15.622 \\
\hline 580.762 & 15.841 & 15.552 & 14.719 & 14.947 & 15.971 & 15.645 & 15.650 & 15.528 & 14.911 & 15.024 & 15.813 & 15.632 \\
\hline 580.773 & 15.910 & 15.511 & 14.792 & 14.967 & 15.966 & 15.608 & 15.668 & 15.531 & 14.990 & 15.017 & 15.889 & 15.582 \\
\hline 580.793 & 15.928 & 15.537 & 14.900 & 15.017 & 15.848 & 15.592 & 15.712 & 15.585 & 15.093 & 15.047 & 15.847 & 15.513 \\
\hline 580.810 & 15.934 & 15.570 & 15.026 & 15.066 & & 15.268 & 15.672 & 15.567 & 15.195 & 15.118 & 15.884 & 15.567 \\
\hline 580.816 & 15.943 & 15.548 & 15.056 & 15.081 & 14.909 & 15.176 & 15.649 & 15.569 & 15.231 & 15.139 & 15.930 & 15.581 \\
\hline 580.826 & 15.920 & 15.563 & 15.123 & 15.096 & 14.537 & 14.963 & 15.648 & 15.550 & 15.272 & 15.137 & 15.945 & 15.695 \\
\hline 580.837 & 15.911 & 15.575 & 15.178 & 15.121 & 14.441 & 14.877 & 15.610 & 15.561 & 15.302 & 15.139 & 15.981 & 15.600 \\
\hline 580.855 & 15.894 & 15.570 & 15.262 & 15.152 & 14.520 & 14.926 & 15.475 & 15.474 & 15.415 & 15.181 & 15.844 & 15.514 \\
\hline 581.683 & 15.825 & 15.564 & & 15.328 & 15.943 & 15.653 & 15.655 & 15.405 & 16.101 & 15.542 & 15.905 & 25.152 \\
\hline 581.701 & 15.936 & 15.562 & 15.855 & 15.426 & 15.929 & 15.628 & 15.652 & 15.531 & 15.929 & 15.545 & 16.041 & 15.841 \\
\hline 581.719 & & 15.528 & & 15.516 & & 15.540 & & & & 15.619 & & 15.504 \\
\hline 581.739 & 15.930 & 15.570 & 15.901 & 15.519 & 15.607 & 15.514 & 15.619 & 15.556 & 15.994 & 15.541 & 15.985 & 15.525 \\
\hline 581.756 & 15.905 & 15.608 & 15.963 & 15.621 & 15.000 & 15.030 & 15.512 & 15.452 & 15.913 & 15.550 & 15.837 & 15.620 \\
\hline 581.774 & & 15.574 & 15.919 & 15.562 & 14.443 & 14.897 & 15.401 & 15.356 & 15.595 & 15.165 & 15.815 & 15.525 \\
\hline 581.790 & 15.898 & 15.566 & 15.857 & 15.471 & & & 15.051 & 15.260 & 15.060 & 15.027 & 15.772 & 15.478 \\
\hline 581.813 & 15.869 & 15.580 & 15.274 & 15.158 & & 15.032 & 15.047 & 15.319 & 14.717 & 14.921 & 15.481 & 15.346 \\
\hline 581.823 & 15.880 & 15.581 & 15.000 & 15.052 & 14.805 & 15.060 & 15.017 & 15.305 & 14.721 & 14.933 & 15.250 & 15.245 \\
\hline 581.839 & 15.934 & 15.646 & 14.712 & 14.930 & 14.958 & 15.122 & 15.001 & 15.345 & 14.829 & 14.966 & 14.887 & 15.087 \\
\hline 581.857 & 15.987 & 15.629 & 14.704 & 14.921 & 15.129 & 15.177 & 15.098 & 15.285 & 14.950 & 15.053 & 14.694 & 14.975 \\
\hline 581.865 & 15.915 & 15.620 & 14.740 & 14.943 & 15.119 & 15.249 & 15.097 & 15.319 & 15.006 & 15.101 & 14.721 & 15.060 \\
\hline 581.875 & 15.706 & 15.438 & 14.801 & 14.995 & & & 15.151 & 15.358 & 15.064 & 15.086 & 14.797 & 15.023 \\
\hline 581.885 & 15.231 & 15.212 & 14.874 & 15.013 & & & 15.223 & 15.350 & 15.134 & 15.065 & 14.848 & 15.079 \\
\hline 581.910 & 14.387 & 14.855 & 15.123 & 15.054 & & & 15.346 & 15.403 & 15.354 & 15.138 & 15.019 & 15.115 \\
\hline 582.656 & 15.921 & 15.573 & 15.839 & 15.390 & 15.907 & 15.668 & 15.446 & 15.499 & 15.829 & 15.353 & 15.853 & 15.563 \\
\hline 582.663 & 15.912 & 15.555 & 15.922 & 15.434 & 15.832 & 15.620 & 15.377 & 15.586 & 15.838 & 15.392 & 15.849 & 15.700 \\
\hline 582.671 & 15.910 & 15.581 & 15.893 & 15.405 & 15.740 & 15.538 & 15.244 & 15.352 & 15.874 & 15.354 & 15.875 & 15.502 \\
\hline 582.690 & 15.911 & 15.534 & 15.879 & 15.469 & 15.271 & 15.294 & 15.034 & 15.242 & 15.861 & 15.432 & 15.882 & 15.502 \\
\hline 582.706 & 15.962 & 15.476 & 15.914 & 15.438 & 14.614 & 14.669 & 15.080 & 15.262 & 15.917 & 15.497 & 15.854 & 15.604 \\
\hline 582.721 & 15.969 & 15.510 & 15.830 & 15.383 & & 14.915 & 15.057 & 15.197 & 15.961 & 15.447 & 15.748 & 15.465 \\
\hline 582.764 & & 15.687 & & 15.434 & & 15.096 & & 15.297 & & 15.655 & & 15.127 \\
\hline 582.774 & & 15.647 & & 15.422 & & 15.106 & & 15.292 & & 15.404 & & 15.018 \\
\hline 582.780 & & 15.658 & & 15.457 & & 15.150 & & 15.299 & & 15.490 & & 15.001 \\
\hline 582.794 & 15.635 & 15.417 & 15.867 & 15.501 & 15.148 & 15.200 & 15.222 & 15.312 & 15.949 & 15.543 & 14.755 & 15.035 \\
\hline 582.819 & 14.446 & 14.869 & 15.850 & 15.500 & & 15.259 & 15.347 & 15.447 & 15.943 & 15.535 & 14.864 & 15.099 \\
\hline 582.835 & 14.422 & 14.876 & 15.942 & 15.554 & & & 15.448 & 15.452 & 15.962 & 15.510 & 14.986 & 15.107 \\
\hline 582.843 & 14.498 & 14.898 & 15.957 & 15.609 & 15.437 & & 15.519 & 15.492 & 15.860 & 15.449 & 15.032 & 15.128 \\
\hline 582.850 & 14.573 & 14.940 & 15.986 & 15.546 & 15.491 & 15.311 & 15.564 & 15.490 & 15.754 & 15.412 & 15.083 & 15.155 \\
\hline 582.858 & 14.651 & 14.997 & 15.992 & 15.554 & 15.518 & 15.372 & 15.592 & 15.515 & 15.517 & 15.341 & 15.129 & 15.121 \\
\hline 582.865 & 14.710 & 15.011 & 15.980 & 15.595 & 15.553 & 15.365 & 15.625 & 15.504 & 15.274 & 15.216 & 15.191 & 15.171 \\
\hline
\end{tabular}

dwarfs that define the Thuan-Gunn system and also serve as spectrophotometric standards. Thus $B-i=0.40$ mag corresponds to $T_{\text {eff }}=6000 \mathrm{~K}$ and $\log (g)=3.7$ (at the metallicity corresponding to the subdwarfs of $Z / Z_{0}=1 / 100$ ). This defines our derivation of $T_{\text {eff }}(\phi)$ assuming the surface gravity is known as a function of phase. We begin by assuming that $g(\phi)$ varies as a result of the variation of $R(\phi)$ only (ignoring nongravitational acceleration). (This is justified in part by considering the relative magnitude of the two accelerations as a function of phase.) We can then assume a mass of $0.6 M_{\odot}$ and take the $R(\phi)$ found by the previous analyses of field RR Lyrae variables, specifically that of Manduca et al. (1981), to provide an initial guess for $g(\phi)$. Thus $T_{\text {eff }}(\phi)$ is completely specified.

We proceed by assuming that the calibration of absolute flux from above Earth's atmosphere by Oke and Gunn (1983) is correct. Using $8100 \AA$ as the effective wavelength for the $i$ filter, we deduce from each observed $i$ magnitude corrected for interstellar absorption the observed flux $f(i)$ in units of ergs $\mathrm{s}^{-1}$ $\mathrm{cm}^{-2} \mathrm{~Hz}^{-1}$. We assume that the emitted flux at $8050 \AA\left(F_{8050}\right)$ of the Kurucz grid of (static) model stellar atmospheres is correct. Thus $F_{8050}$ is parameterized as a function of $T_{\text {eff }}$ and $g$ for the models with metallicity one-tenth the solar value. We then solve directly for the angular diameter,

$$
\theta(\phi)=2 \sqrt{\frac{f(8100)}{\pi F_{8050}\left[T_{\text {eff }}(\phi), g(\phi)\right]}}
$$

where the factor of $\pi$ converts Kurucz's tabulated fluxes to astrophysical fluxes. Thus each pair of $B, i$ magnitudes is transformed into a point on the photometric $\theta(\phi)$ relationship. This calculation is done only once, as the derived $\theta(\phi)$ is completely specified by the observational data, the grid of model atmospheres, and the absolute flux conversion factors.

We can verify the accuracy of this analysis by considering stars with known angular diameters. There are two stars for which $\theta$ was measured by Hanbury Brown, Davis, and Allen (1974) which lie in the $T_{\text {eff }}$ regime under consideration here. Unfortunately, neither of those has absolute spectrophotometry nor near-infrared $i$ photometry, so that $T_{\text {eff }}$ for these test cases cannot be derived from their $B-i$ colors. However, if we use, for example, the results of Dreiling and Bell (1980) for the $T_{\text {eff }}$ and surface gravity of $\alpha$ Lyrae, assuming solar metallicity, to predict the star's emitted flux and take its observed flux at $8100 \AA$ from Oke and Gunn (1983), we deduce $\theta($ Vega $)=$ 


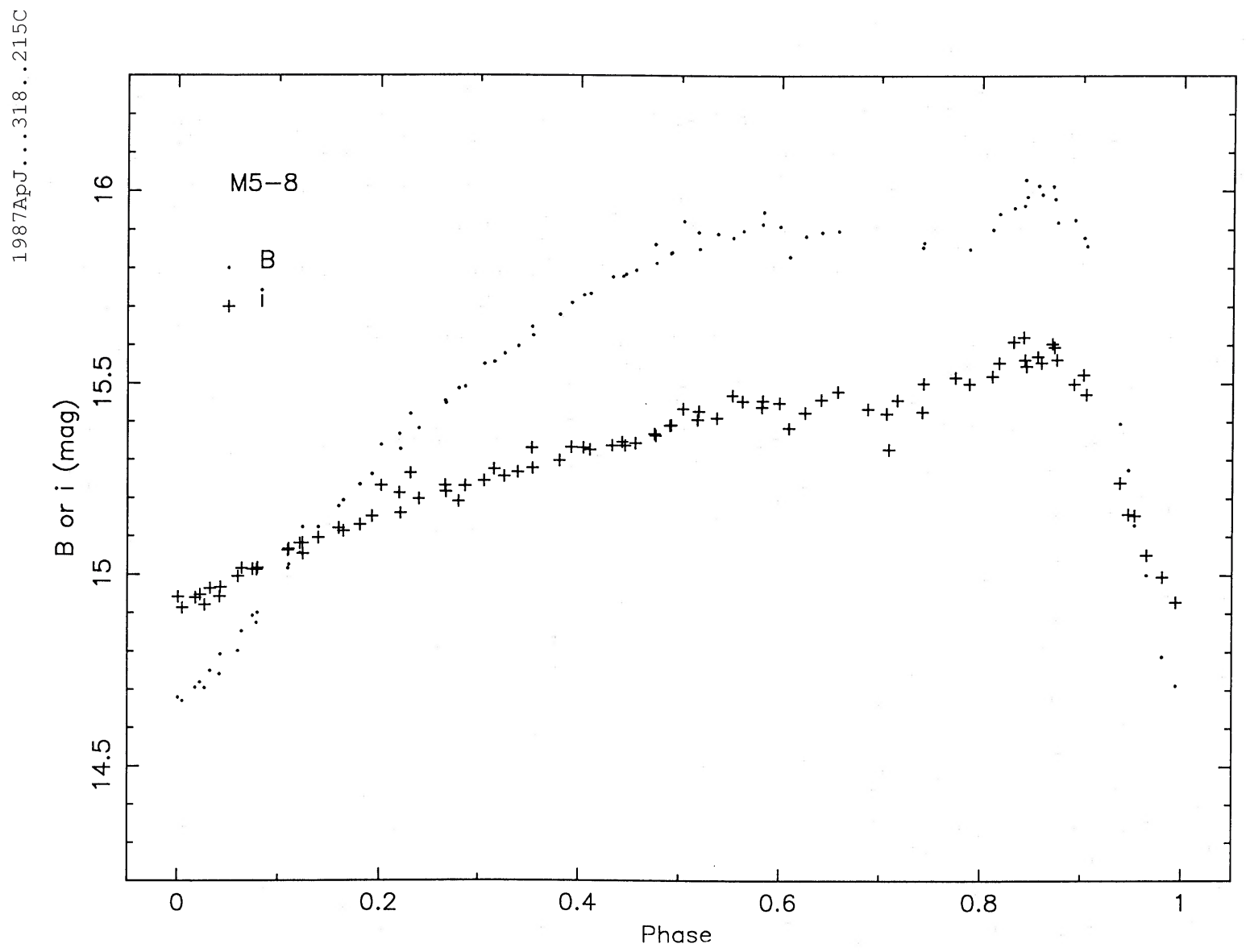

FIG. $2 a$

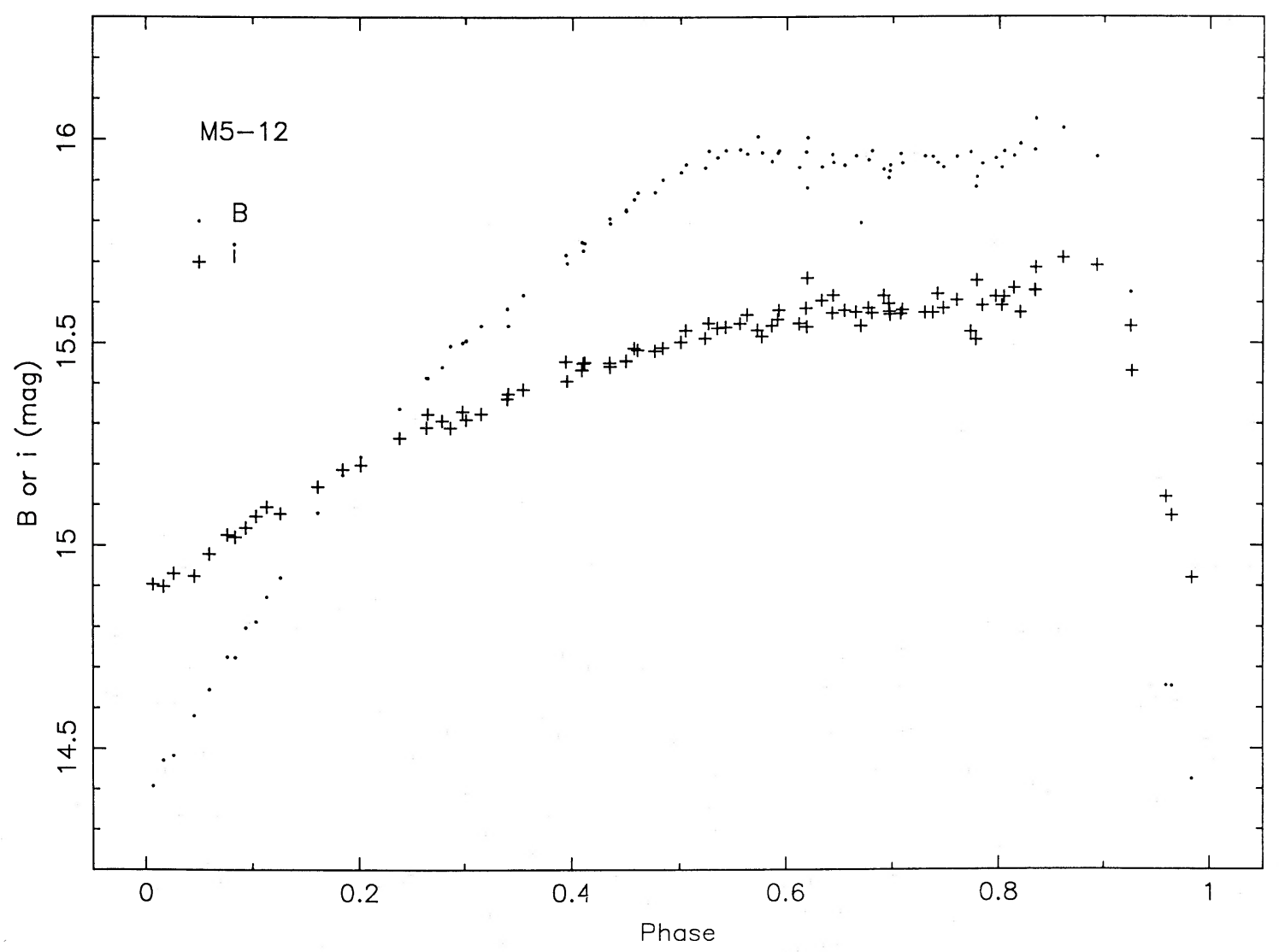

Fig. $2 b$

FIG. 2.-The $B$ (points) and $i$ (crosses) light curves for the four RR Lyrae variables in M5 are shown

224

(C) American Astronomical Society - Provided by the NASA Astrophysics Data System 


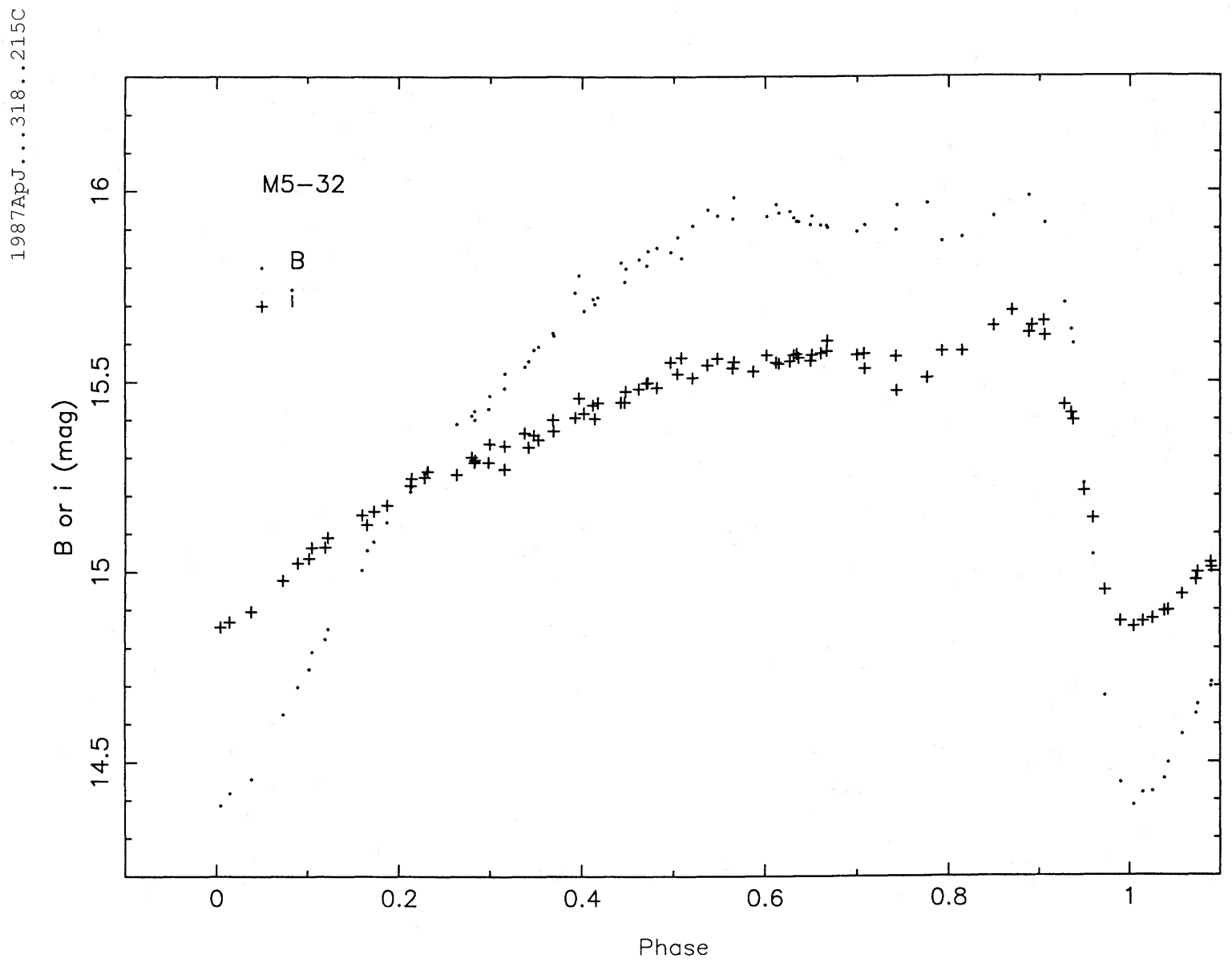

FIG. $2 c$

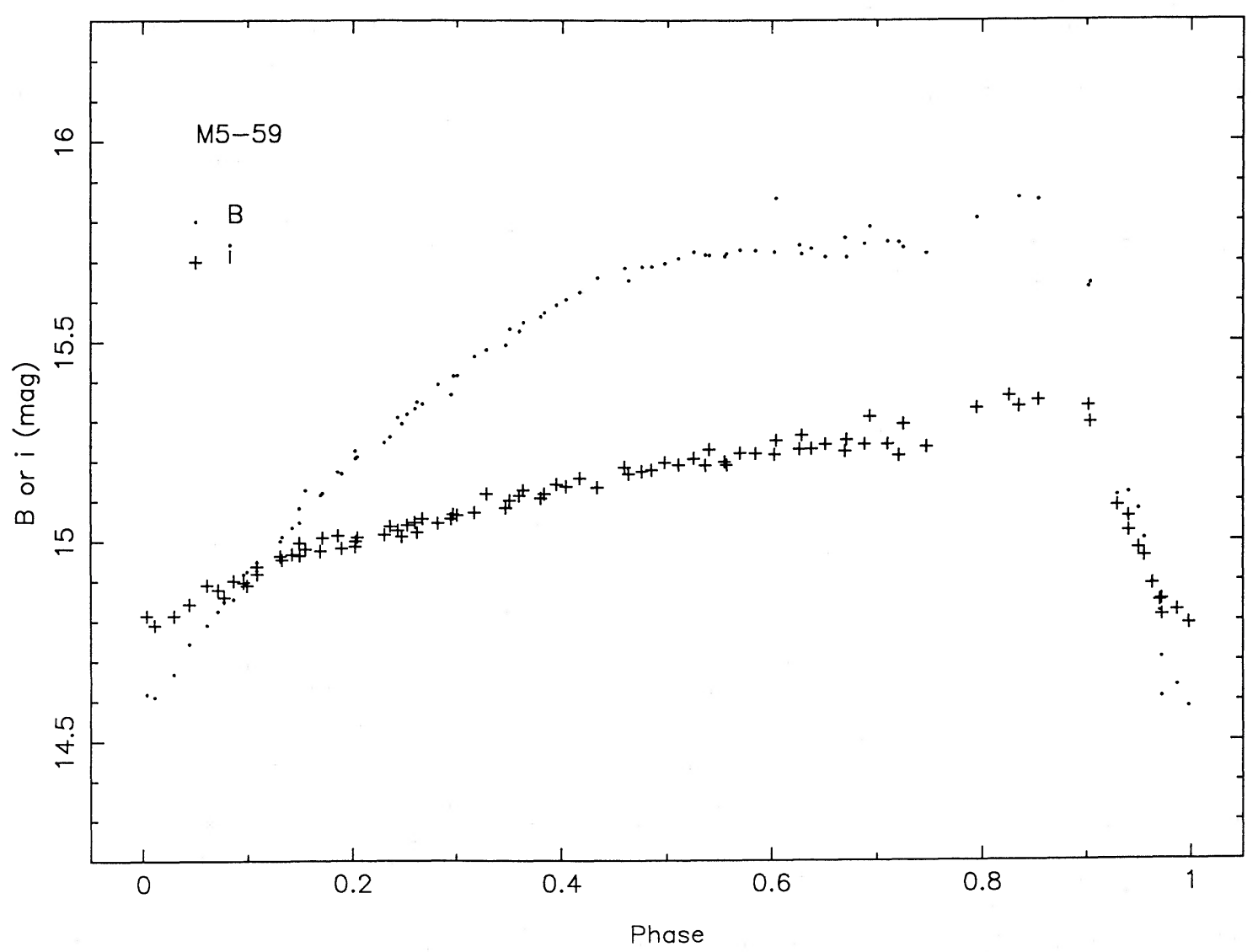

Fig. $2 d$

225

(C) American Astronomical Society - Provided by the NASA Astrophysics Data System 
0 .00320, equal to the observed value of $3 . .24 \pm 0$ ".07 $\times 10^{-3}$ (Hanbury Brown, Davis, and Allen 1974) to within the uncertainty of the measurement.

We now vary the values adopted for the distance and radius at $\phi=0$ until the two $\theta-\phi$ relationships are in best coincidence. Several fitting routines were written for this purpose. The best value of $R(\phi=0)$ for a given distance is found by minimizing the sum

$$
\sum_{i=1}^{N}\left[\theta(\text { phot })_{i}-\theta\left(v_{r}\right)\right]
$$

over each of the $N$ pairs of $B, i$ magnitudes with both terms evaluated at the phase corresponding to the $i$ th pair. Let us denote the derived best value of $R(\theta=0)$ for a given distance as $R_{D}$. Then we calculate a series of sums for various choices of the distance $D$. These sums are identical to the first set except that for each choice of $D$ the appropriate value of $R_{D}$ is adopted and also each term in the sum written above is replaced by its absolute value. The best value of the distance is that for which this second sum is minimized. Both of these sums are evaluated only over a certain range in phase within which our assumptions are valid. Ideally that is the whole cycle but as described subsequently the interval actually used was substantially less. Once this first pass is completed to derive final value of distance we should then iterate to correct our assumed surface gravity- $\phi$ relationship, but the problems described in $\S \mathrm{IV} a$ were so much more serious that this was in fact never done.

\section{b) Application to the Present Data}

When this method is applied to our data, several practical problems manifest their existence. The photometric data set contains only about 90 pairs of points. Figure 2 illustrates the light curves $B$ or $i$ as a function of $\phi$ and we notice that there are several discrepant points in each light curve, most of which are from the last two rather cloudy nights. These points were smoothed onto the mean magnitude-color relationship. (This was done individually for each color, so that it is not necessarily true that the same points were smoothed in each color. The number of points thus treated ranged between two and seven. Typical corrections in magnitude were $\sim 0.05 \mathrm{mag}$ for $B$ or $i$.) Furthermore there are also up to four observations from the two cloudy nights with no $B$ magnitudes, only $i$ measurements. If for a particular star these fell into a phase interval where there were few points, they were added in with a smoothed $B$ magnitude; otherwise they were ignored.

\section{RESULTS}

\section{a) Distances and Radii}

The procedures described in $\S$ III have been applied to the data we obtained for the RR Lyraes in M5. Figure 3 shows the angular diameter-phase relationships used to determine the distance and mean radius for each of the variables. The crosses are from the individual $B, i$ magnitude pairs and their locations are independent of the assumed distances and mean radii for the RR Lyrae variables. The solid curve is from the integration of the radial velocities using the distance which gives the best fit and the appropriate value of $R_{D}$. The filled squares represent the $\theta-\phi$ relationship for the smallest distance which gave an acceptable fit, and the filled circles represent that for the largest possible distance which gave acceptable results. Figure $3 a$ includes only those phases where $T_{\text {eff }}$ is not more than $1000 \mathrm{~K}$ above the minimum value of $T_{\text {eff }}$. This removes an interval averaging 0.30 cycles centered on maximum visual light (minimum radius, maximum $T_{\text {eff }}$, maximum nongravitational acceleration). The resulting values of distance, mean radius, intensity mean absolute $V$ magnitude, and distance modulus (the last two quantities both corrected for absorption) are listed in Table 4 for the best fit, as well as for the largest and smallest distances which yielded acceptable fits. These fits are in fact determined over a still smaller interval in phase as discussed later in this section. We also list the full amplitude of the radius variations over the period derived from integrating the radial velocity curve, which is independent of choice of distance but depends on the choice of projection factor $p$. The mean of the absorption corrected intensity mean $V$ magnitudes for the four RR Lyrae stars in M5 is $+1.05 \mathrm{mag}$, with a range of $+0.15,-0.25 \mathrm{mag}$ derived from averaging the limits of the acceptable fits given in Table 4 . This is $0.25 \mathrm{mag}$ fainter than

TABLE 4

Distances and Mean Radil for M5 RR Lyrae Stars

\begin{tabular}{|c|c|c|c|c|c|}
\hline Star & $\begin{array}{l}\text { Distance } \\
(\mathrm{kpc})\end{array}$ & $\begin{array}{c}\text { Mean Radius } \\
(\mathrm{km})\end{array}$ & $\begin{array}{c}\text { Intensity Mean } \\
V_{0}\end{array}$ & $(m-M)_{0}$ & $\begin{array}{c}\Delta R \\
(\mathrm{~km})\end{array}$ \\
\hline \multicolumn{6}{|c|}{ A. Best Fit } \\
\hline M5-8. & 5.83 & $3.07 \mathrm{E} 6$ & +1.21 & 13.83 & 533,600 \\
\hline M5-12 ......... & 5.83 & $2.79 \mathrm{E} 6$ & +1.20 & 13.83 & 504,000 \\
\hline M5-32 ......... & 6.48 & $3.07 \mathrm{E} 6$ & +0.95 & 14.06 & 501,700 \\
\hline M5-59......... & 6.16 & $3.66 \mathrm{E} 6$ & +0.93 & 13.95 & 470,300 \\
\hline \multicolumn{6}{|c|}{ B. Smallest Acceptable Distance } \\
\hline M5-8 $\ldots \ldots \ldots$ & 5.51 & $2.89 \mathrm{E} 6$ & +1.33 & 13.71 & \\
\hline M5-12 ........ & 5.51 & $2.63 \mathrm{E} 6$ & +1.32 & 13.71 & \\
\hline M5-32 .......... & 5.83 & $2.74 \mathrm{E} 6$ & +1.18 & 13.83 & \\
\hline M5-59......... & 5.51 & $3.29 \mathrm{E} 6$ & +1.17 & 13.71 & \\
\hline \multicolumn{6}{|c|}{ C. Largest Acceptable Distance } \\
\hline M5-8 $\ldots \ldots \ldots$ & 6.48 & $3.41 \mathrm{E} 6$ & +0.98 & 14.06 & \\
\hline M5-12 $\ldots \ldots \ldots \ldots$ & 6.48 & $3.11 \mathrm{E} 6$ & +0.97 & 14.06 & \\
\hline M5-32 .......... & 7.12 & $3.36 \mathrm{E} 6$ & +0.75 & 14.26 & \\
\hline M5-59......... & 6.80 & 4.07E6 & +0.72 & 14.16 & \\
\hline
\end{tabular}




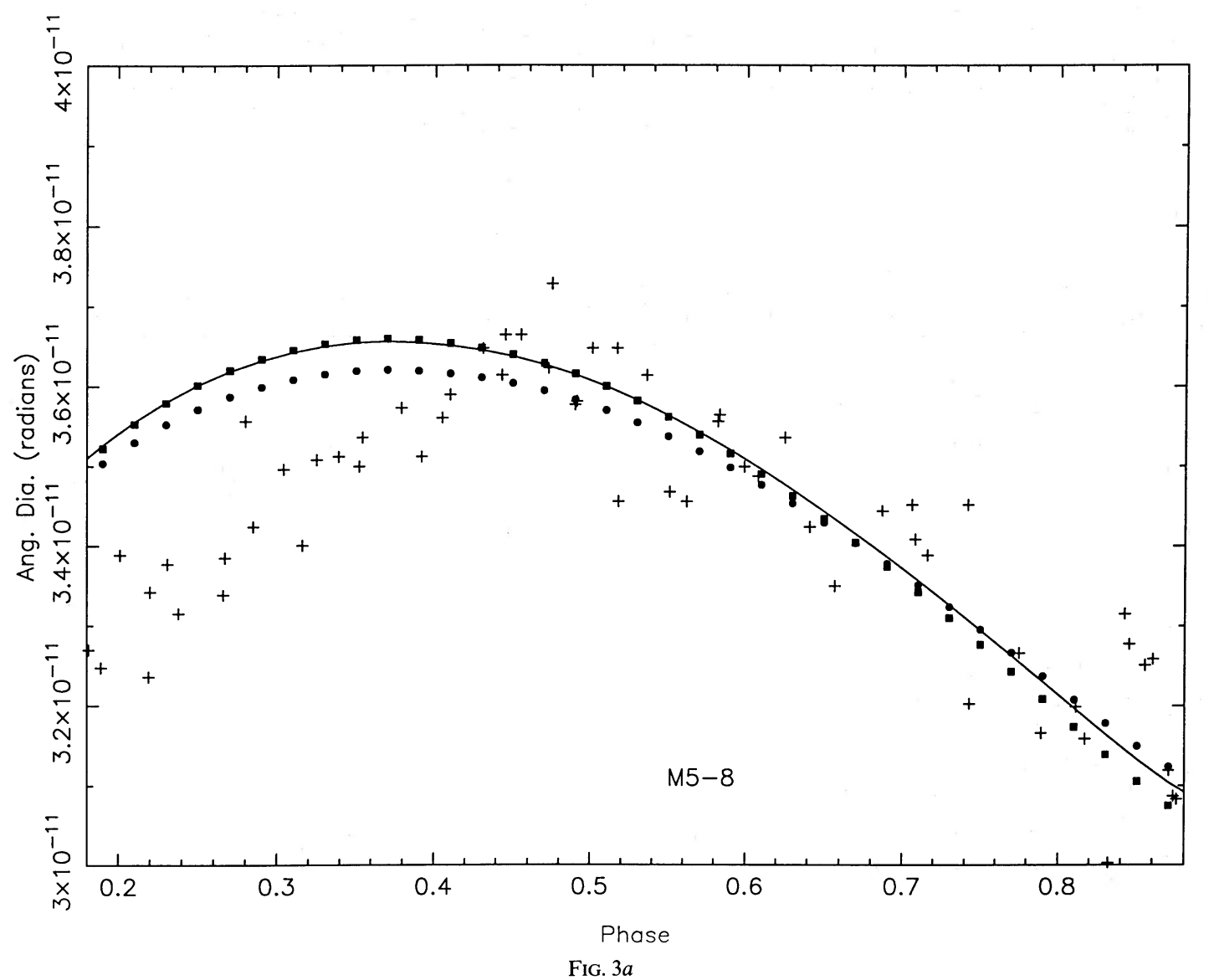

FIG. 3. - The angular diameter deduced from the photometric and from the spectroscopic data sets is plotted as a function of phase for the RR Lyrae star M5-8. The crosses represent the values of $\theta$ derived from each pair of $B$ and $i$ measurements. The solid curve is from the integration of the radial velocity curve with the choice of distance and the appropriate mean radius which gave the best fit between the two $\theta-\phi$ relationships. The filled squares represent the $\theta-\phi$ relationship for the smallest distance judged acceptable for this variable, while the filled circles illustrate the $\theta-\phi$ relationship for the largest distance judged acceptable for this star. (b) Same as $(a)$ for M5-12. (c) Same as $(a)$ for M5-32. (d) Same as $(a)$ for M5-59.

was adopted by Sandage (1982) for M5, resulting in a distance which is $89 \%$ as large as that of Sandage.

In spite of our effort at smoothing the photometry, the errors in our limited set of $B$ and $i$ magnitudes produce a significant scatter in the resulting $\theta(\phi)$ relationship. This directly produces the rather wide range of fits (hence of distances) which were judged acceptable. The scatter arises in part because for each star there are intervals in phase which contain a number of points which is too small to allow us to judge whether or not there are poor measurements included therein and how to properly smooth them. Thus in some cases the formal fitting procedure given above for determining the best disance fails due to the nonrandom distribution of photometrically determined $\theta(\phi)$ values about the $\theta(\phi)$ curve deduced from the radial velocities.

The major problem apparent from Figure 3 is the asymmetry of the photometric $\theta(\phi)$ curve compared to that from the radial velocities, which manifests itself as systematic deviation which increases progressively as one approaches maximum light $(\phi=0)$. This cannot be a phase shift in the data or a period error, as it has approximately the same sign and magnitude in all four variables, and the photometry and radial velocity data were obtained within a relatively short time interval for three of the four stars. This apparent asymmetry problem is seen in similar studies of field RR Lyrae variables (see Carney and Latham 1984; Jones et al. 1987a, b). It is believed to be due to the effects of shocks lingering beyond the interval in phase around maximum light which we already omitted. If that is the case, at $\phi \approx 0.20$, overestimates of $T_{\text {eff }}$ by about $250 \mathrm{~K}$ from our $B-i$ colors are required to bring the two $\theta(\phi)$ relationships into coincidence. This corresponds to an excessively blue $B-i$ color by about $0.13 \mathrm{mag}$, presumably arising largely from an excess flux in the blue spectral region due to perturbation of the atmosphere by shocks. The apparent overestimate of $T_{\text {eff }}$ decreases progressively toward larger phases. Therefore in our distance determinations, the interval in phase used in the fitting was further restricted to 0.43 to 0.88 cycles for M5-8, 0.48 to 0.88 cycles for M5-12, 0.46 to 0.92 cycles for M5-32, and 0.45 to 0.88 cycles for M5-59. The fits determined over these intervals are those listed in Table 4 and plotted in Figure 3.

At this point, we recall that the range of distances listed for each star in Table 4 includes only the uncertainties arising from the limited available photometry and the small range in phase judged useful for matching the $\theta-\phi$ curves. Although we cannot assess the systematic error that might result from the effects of the apparent phasing problem in fact lingering toward even later phases than the larger interval we have already discarded, we note that any such systematic error is likely to underestimate the angular diameter from the $B, i$ photometry and hence overestimate the distance. Since our 


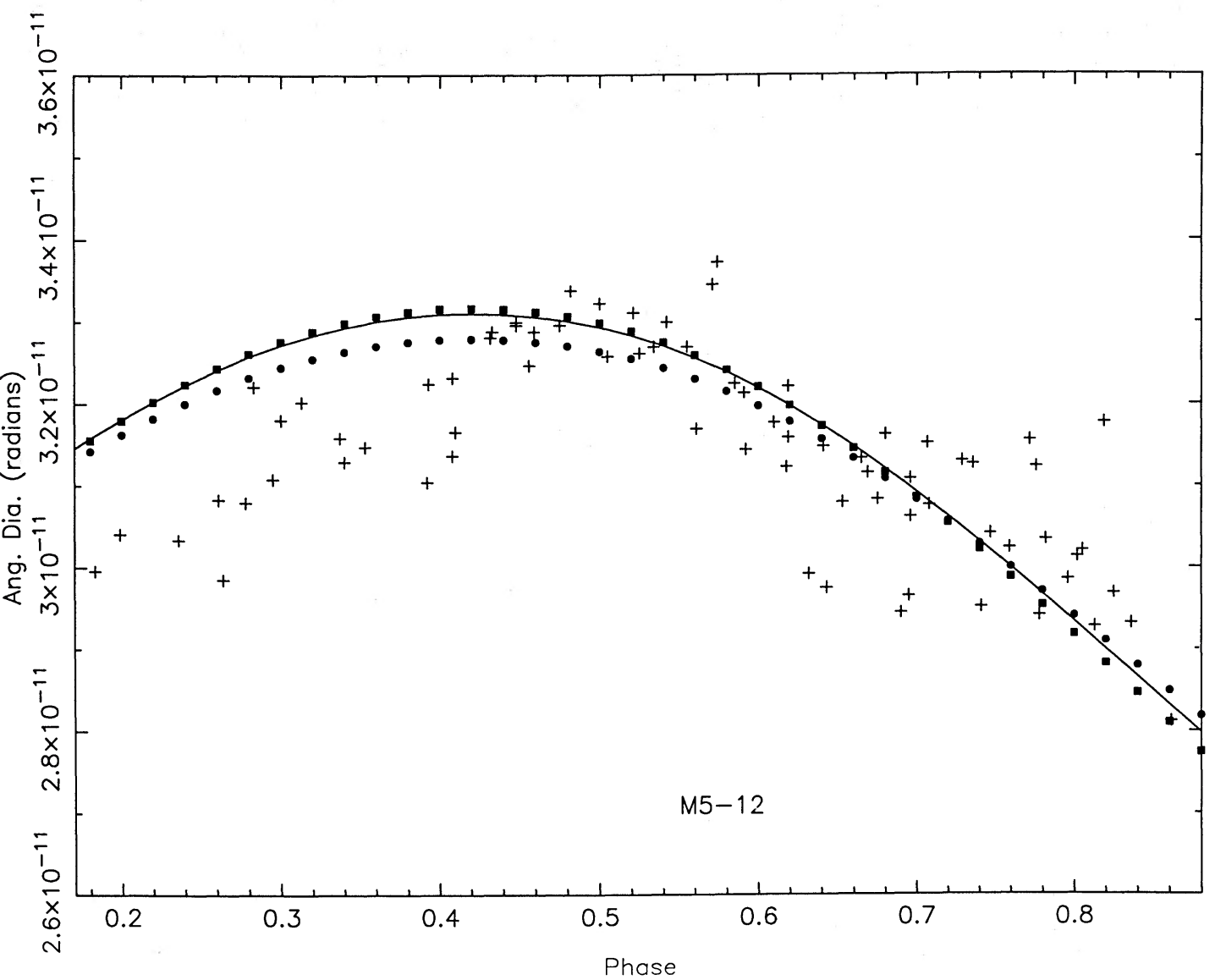

FIG. $3 b$

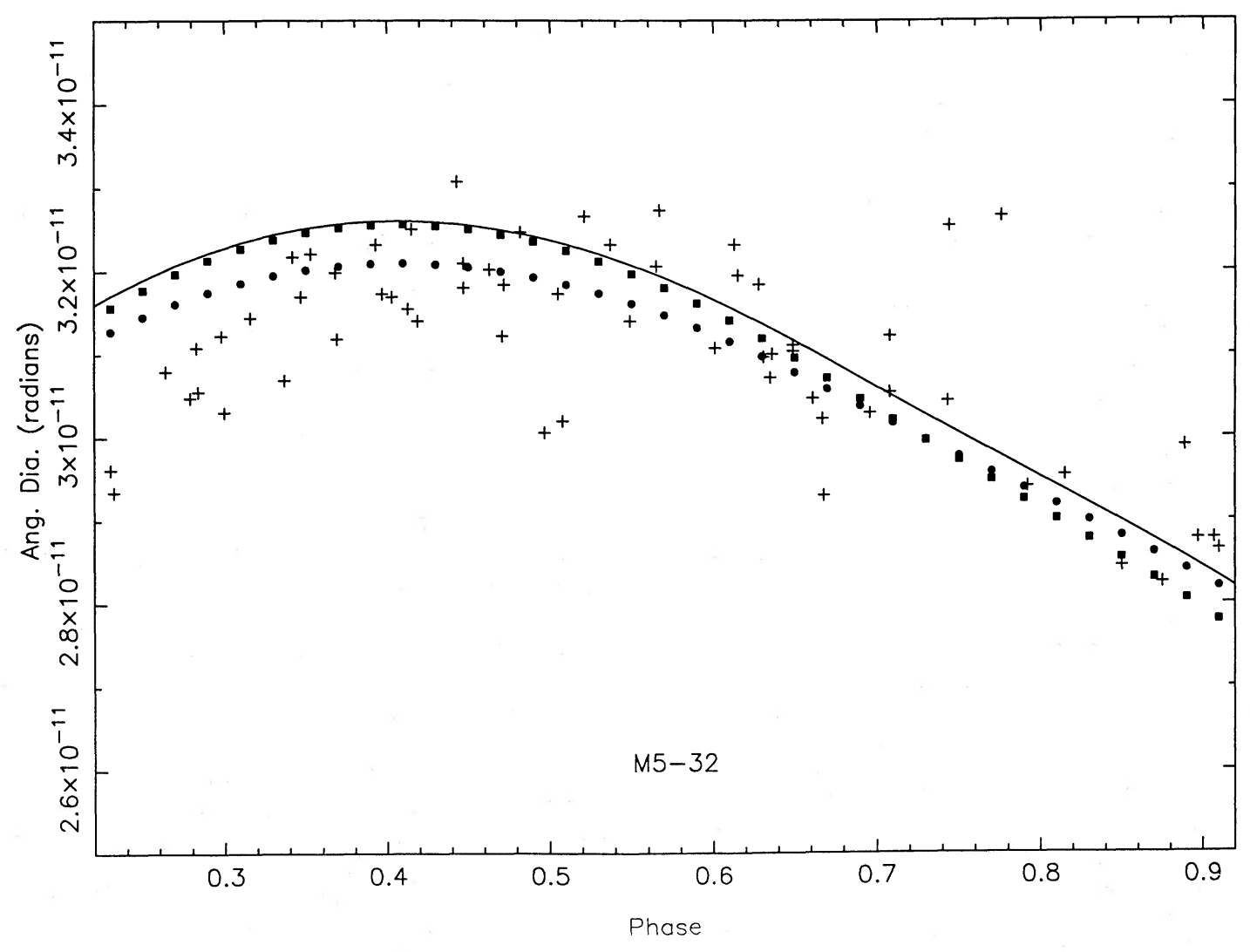

FIG. $3 c$ 


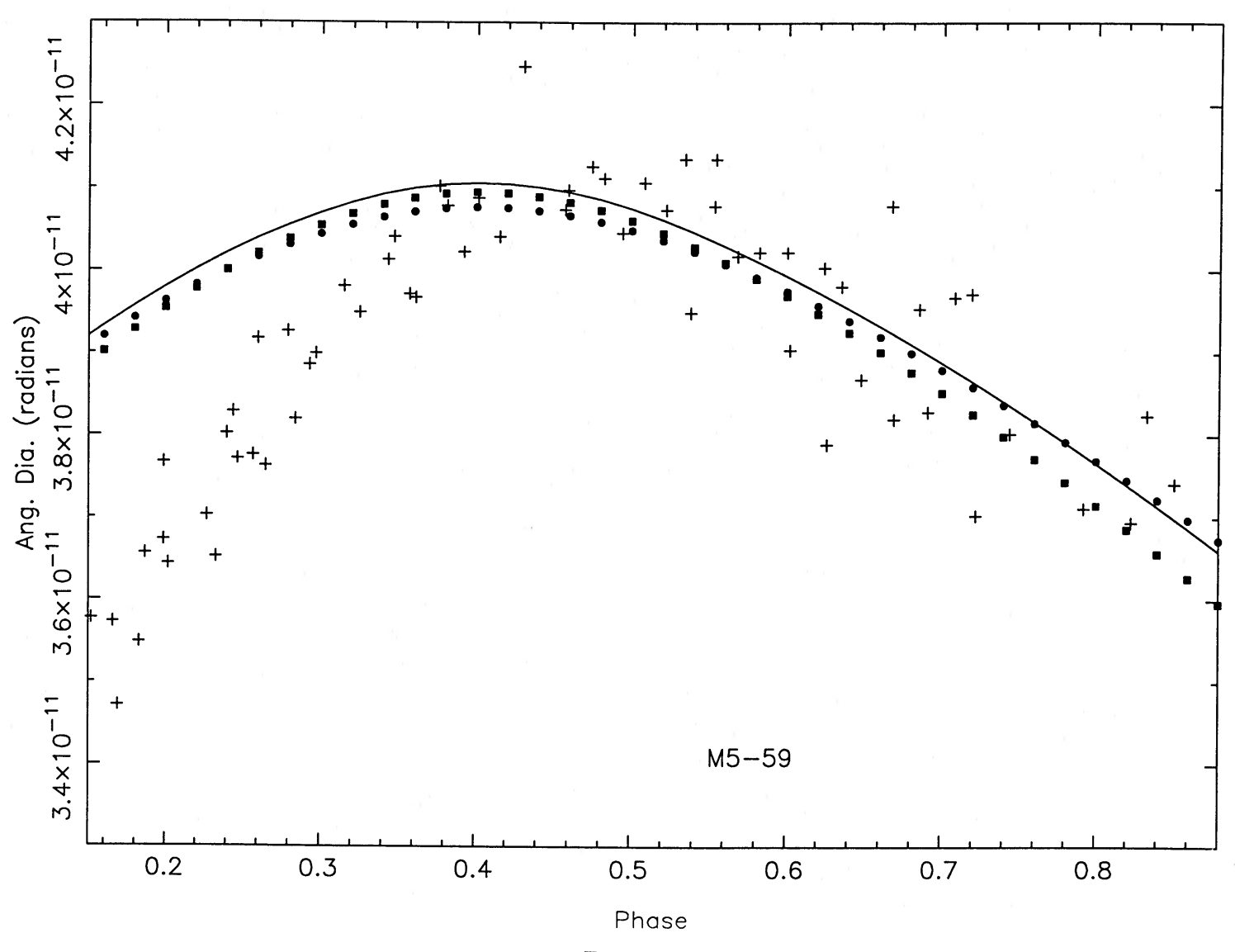

FIG. $3 d$

distance to M5 is already quite small, we feel that we have eliminated a sufficiently large interval in phase to remove any error arising from the apparent asymmetry, although this remains to be verified by further work planned for the 1986 observing season.

We now justify our assertion that several simplifications and assumptions which we glossed over earlier do in fact contribute only negligible errors to our distances. The uncertainty in the absolute calibration quoted by Oke and Gunn (1983) is 5\% in the flux. The error made by neglecting the difference between $8100 \AA$ and $8050 \AA$ in evaluating the absolute flux is less than $0.005 \mathrm{mag}$, based on the tables of Oke and Gunn. The error made by ignoring the difference between the metallicity of M5 at about -1.5 dex and the models used (at -1.0 dex with respect to the Sun) is less than $4 \%$ in the absolute flux at 8050 $\AA$. For a fixed $B-i$ color, the change in $T_{\text {eff }}$ when the metallicity is changed from 0.1 to 0.01 that of the Sun is less than 100 $\mathrm{K}$, which in turn corresponds to an error in the absolute flux at $8050 \AA$ of less than $5 \%$. Since the flux is proportional to $D^{-2}$, it is clear that the uncertainty in the distance we derive for the M5 RR Lyraes is dominated by the small regime in phase deemed acceptable and by the limited photometry within that regime of phase and the uncertainties in the photometry itself. Once the problems in the photometry are resolved, the small effects described above must of course be taken into account.

\section{b) Comparison with Previous Applications of Baade-Wesselink Techniques to RR Lyrae Stars}

The many previous studies of field RR Lyraes were listed in $\S$ I. Without the extensive work of Carney and Latham (1984) and Jones et al. $(1987 a, b)$, we would be quite stumped by the apparent asymmetries in the light curves. Their work has demonstrated that these asymmetries become less significant when colors at redder wavelengths are used. In addition, they have shown that blue colors give results consistent with redder colors over only the latter part of the cycle, and we have used their results to guide us in choosing to reject the part of the cycle closest to maximum light when we fit for distance and mean radius. They too obtain magnitudes for the three RR Lyrae field stars which they have analyzed that are rather faint, averaging about +0.85 for magnitude-averaged, absorptioncorrected absolute $V$ magnitudes. Our only criticism of the analysis of Carney and collaborators is that since they do not use an automatic fitting program, but merely adjust radii and distances until a visually satisfying fit is obtained, it may be that their estimates of the uncertainty of their distances and absolute mean magnitudes due just to the fitting procedure are actually too small.

It is rather more difficult to compare our results to those of the CORAVEL group (Burki and Meylan 1986a, $b$ and refer- 
ences therein), who in general obtain brighter absolute mean magnitudes for the two field RR Lyrae stars for which they have published an analysis. This is because the most critical step in any Baade-Wesselink method is the determination of $T_{\text {eff }}$ as a function of phase. Although they have multicolor photometry in the Geneva system, most of their colors are in the blue spectral region, and they have not published the details of their transformation between color and $T_{\text {eff }}$. They have noted the presence of excess ultraviolet light near maximum light in their analysis of RR Cet. The British group, whose progress is reviewed in Jameson (1986), used visual and infrared photometry on four field RR Lyrae stars to derive a strong metallicity dependence in the absolute mean $V$ magnitudes, which was not found by Carney and his collaborators in a smaller sample of stars. Their temperature determinations are derived by assuming a linear relationship between color and $\log \left(T_{\text {eff }}\right)$ whose coefficients are empirically determined from the observations of the RR Lyrae variable itself. This is a variation of the method of Balona (1977). A detailed critique of the approaches used by both of these groups can be found in Jones et al. $(1987 b)$.

It is clear that no such result from an application of the Baade-Wesselink method to RR Lyrae stars can be fully trusted until the extent of the flux discrepancy is mapped as a function of phase and wavelength. The critical assumption that a series of static model atmospheres may be applied to successive phases of a pulsating variable star with a short period is made in some form by all groups working in the field. This gives rise to the major uncertainty affecting $R R$ Lyrae distances today. Hopefully we will obtain the necessary data during the coming year to satisfy ourselves that within some limits of phase and wavelength Baade-Wesselink techniques can in fact be used to determine accurate distances for $R R$ Lyrae variables.

\section{SUMMARY}

The observational material necessary for the application of the Baade-Wesselink method of determining distances has been obtained for four RR Lyrae variables in the globular cluster M5. We have measured radial velocity-phase curves from spectra taken with the 200 inch Hale telescope at Palomar Observatory. We have between 28 and 56 independent values per star with an accuracy no worse than $\pm 4 \mathrm{~km}$ $\mathrm{s}^{-1}$ per radial velocity. Light curves for the four RR Lyrae stars have been obtained using an imaging CCD system at the 60 inch telescope at Palomar Mountain. We took short exposure frames through a Johnson $B$ and a near-infrared $i$ filter. The photometric and the spectroscopic data sets were obtained within a 6 week interval in 1986 April-May, except for the RR Lyrae star M5-32, which was also observed spectroscopically in 1985. About 90 pairs of $B$ and $i$ magnitudes are available for each star. There are also light curves for six additional RR Lyrae variables in M5 that fell within the area covered by the two CCD fields. These light curves have been transformed to $V$ magnitudes, then integrated to yield mean and intensity mean $V$ magnitudes for each variable.

The method of analysis which has been developed for the data requires matching the angular diameter-phase relationship determined from the spectroscopic and from the photo- metric data sets. The treatment of the spectroscopic data is straightforward. The $v_{r}-\phi$ relationship is integrated using a projection factor to convert between radial velocity and pulsational velocity of 1.31 . The mean velocity $\left(v_{\gamma}\right)$ of each of the four RR Lyrae stars is within $4 \mathrm{~km} \mathrm{~s}^{-1}$ of the measured radial velocity of M5 itself as compiled by Webbink (1981). If one assumes a value for the distance to M5 and a value of the stellar radius at $\phi=0$, the spectroscopic $\theta-\phi$ relationship is easily obtained. The photometric data is treated by using an effective wavelength for the $B$ and the $i$ filters, assuming that the absolute calibration of observed flux from the bright spectrophotometric standards by Oke and Gunn (1983) is correct, and transforming the observed magnitude at $i$ into a flux above Earth's atmosphere. This is compared with an emitted flux per unit of radiating area from the appropriate model stellar atmospheres of the grid by Kurucz (1979) at the effective wavelength of the $i$ filter. The effective temperature is deduced from the $B, i$ colors (with the metallicity and gravity dependence taken into account as discussed in detail in $\S$ III $a$ ).

The distance and mean radius are derived by fitting algorithms which match the spectroscopically and photometrically derived $\theta-\phi$ curves. Averaging the results from the four M5 RR Lyraes analyzed in detail, this procedure yields an intensity mean, interstellar absorption-corrected $V$ magnitude of +1.05 mag. Because of the limited photometry, its limited accuracy (two of the nights used were cloudy, and sometimes data from these nights fell in critical regions of phase), and the limited region of phase that could be used to deduce the distance, the fitting errors alone permit values up to +0.15 mag fainter or -0.25 mag brighter. This does not include any systematic errors or uncertainties arising from the method of analysis, which are believed to be small compared to the fitting uncertainties, or from the limited region in phase that was used due to asymmetry problems in the light curves. These asymmetry problems which have been seen in other investigations of field RR Lyrae variables utilizing blue colors (see, for example, Carney and Latham 1983), greatly restrict the range of phase that can be used to derive the distances, eliminating a substantial interval in phase beginning just before maximum visual light.

After a review of existing field star Baade-Wesselink analyses, we find that the primary remaining area of concern is the applicability of a set of static stellar atmospheres to a pulsating variable star at a given phase. We plan to test the validity of this procedure to determine the limits of phase and wavelength over which such an approach is accurate. Although we believe that by eliminating a substantial interval in phase and by determining the flux at a near-infrared color we have avoided the worst of these problems, it is nonetheless true that until these limits have been determined, all distances derived from Baade-Wesselink analyses of RR Lyrae stars must be regarded with some degree of skepticism.

This work was initiated under NSF grant AST-8212270. J. G. C. is grateful to the Caltech Recycling Center and to Gaston Araya Machining for financial and other support. G. A. G. was supported during part of the time by a Caltech Summer Undergraduate Research Fellowship. 


\section{REFERENCES}

Abt, H. A., and Biggs, E. S. 1972, Bibliography of Stellar Radial Velocities (New York: Latham Process Co.).

Arp, H. C. 1962, Ap. J., 135, 311

Baade, W. 1926, Astr. Nach., 228, 359.

Bailey, S. I. 1917, Harvard Ann., 78, 103.

Balona, L. A. 1977, M.N.R.A.S., 178, 231

Barnes, T. G., III, and Hawley, S. L. 1986, Ap. J. (Letters), 307, L9.

Bell, R. A., and Oke, J. B. 1986, Ap. J., 307, 253.

Burki, G., and Meylan, G. 1986a, Astr. Ap., 156, 131. 1986 , Astr. Ap., 159, 255

Cacciari, C. 1986, private communication

Carney, B. W. 1980, Ap. J. Suppl., 42, 481.

Carney, B. W., and Latham, D. W. 1984, Ap. J., 278, 241.

Coutts, C., and Sawyer-Hogg, H. 1969, Pub. David Dunlop Obs., Vol. 3, No. 1.

Dreiling, L. A., and Bell, R. A. 1980, Ap. J., 241, 736

Frogel, J. A., Cohen, J. G., and Persson, S. E. 1983, Ap. J., 275, 773.

Graham, J. A. 1984, in IAU Symposium 108, Structure and Evolution of the Magellanic Clouds, ed. S. van den Bergh and K. S. de Boer (Dordrecht: Reidel), p. 207.

Graham, J. A., and Nemec, J. M. 1984, in IAU Symposium 108, Structure and Evolution of the Magellanic Clouds, ed. S. van den Bergh and K. S. de Boer (Dordrecht: Reidel), p. 37

Gunn, J. E., and Griffin, R. 1979, A.J., 84, 752.

Hanbury Brown, R., Davis, J., and Allen, L. R. 1974, M.N.R.A.S., 167, 121.

Hawley, S. L., Jeffreys, W. H., Barnes, T. G., III, and Lai, Wan. 1986, Ap. J., $302,626$.

J. G. CoHEn and G. A. Gordon: 105-24, California Institute of Technology, Pasadena, CA 91125

. 1982, Ap.J., 252, 561.
Hindsley, R. and Bell, R. A. 1986, Pub. A.S.P., 98, 881.

Hogg, H.S. 1973, Pub. David Dunlop Obs., Vol. 3, No. 6.

Jameson, R. F. 1986, Vistas Astr., 29, 17.

Jones, R. V., Carney, B. W., Latham, D. W., and Kurucz, R. L. 1987a, Ap. J., 312, 254.

. $1987 b$, preprint.

Kurucz, R. L. 1979, Ap. J. Suppl., 40, 1.

Longmore, A. J., Fernley, J. A., Jameson, R. F., Sherrington, M. R., and Frank, J. 1985, M.N.R.A.S., 216,873 .

Manduca, A., and Bell, R. A. 1981, Ap. J., 250, 306.

Manduca, A., Bell, R. A., Barnes, T. G., III, Moffett, T. J., and Evans, D. S. 1981, Ap. J., 250, 312.

Oke, J. B. 1966, Ap. J., 145, 468.

Oke, J. B., and Gunn, J. E. 1983, Ap. J., 266, 713

Oort, J. H., and Plaut, L. 1975, Astr. Ap., 41, 71.

Pritchet, C. J., and van den Bergh, S. 1986, preprint. Sandage, A. $1970, A p . J ., 162,841$.

Schneider, D. P., Gunn, J. E., and Hoessel, J. G. 1983, Ap. J., 264, 337.

Strugnell, P., Reid, N., and Murray, C. 1986, M.N.R.A.S., 220, 413.

Thuan, T. X., and Gunn, J. E. 1976, Pub. A.S.P., 88, 543.

Wade, R. A., Hoessel, J. G., Elias, J. H., and Huchra, J. P. 1979, Pub. A.S.P., 91, 35.

Webbink, R. F. 1981, Ap. J. Suppl., 45, 259.

Wesselink, A. J. 1969, M.N.R.A.S., 144, 297.

Zinn, R. J. 1980, Ap. J.Suppl., 42, 19. 